\title{
The boundedness of commutators associated with Schrödinger operators on Herz spaces
}

Pengtao $\mathrm{Li}^{1 *}$ and Xin Wan ${ }^{2}$

*Correspondence: ptli@qdu.edu.cn

'Department of Mathematics, Qingdao University, Qingdao, Shandong 266071, China

Full list of author information is available at the end of the article

\begin{abstract}
Let $L=-\Delta+V$ be a Schrödinger operators on $\mathbb{R}^{n}, n \geq 3$, where the nonnegative potential $V$ belongs to the reverse Hölder class $B_{s}$ for $s>\frac{d}{2}$. Let $T_{\beta}=(-\Delta+V)^{-\beta} V^{\beta}$, $\beta>0$, and $R_{L}=\nabla(-\Delta+V)^{-1 / 2}$ be the Riesz transform associated to $L$. We prove that the operator $T_{\beta}$ and $R_{L}$ are bounded on Herz spaces $\dot{K}_{q}^{\alpha, p}\left(\mathbb{R}^{n}\right)$ and $K_{q}^{\alpha, p}\left(\mathbb{R}^{n}\right)$, respectively. Suppose that $b \in \mathrm{BMO}_{\sigma}(\rho)$, which is larger than $\mathrm{BMO}\left(\mathbb{R}^{n}\right)$. By a maximal estimate, we obtain the boundedness of commutators $\left[b, T_{\beta}\right]$ and $\left[b, R_{L}\right]$ on Herz spaces.
\end{abstract}

MSC: Primary 42B20; 42B35

Keywords: commutator; Herz space; Schrödinger operator

\section{Introduction}

Let $L=-\Delta+V$ be a Schrödinger operators on $\mathbb{R}^{n}, n \geq 3$, where the nonnegative potential $V$ belongs to the reverse Hölder class $B_{s}, s>\frac{n}{2}$. The Riesz transforms and the fractional integrals associated with $L$ have been studied extensively. In 1995, Shen [1] proved the $L^{p}$-boundedness of the operators $(-\Delta+V)^{i \gamma}, \nabla^{2}(-\Delta+V)^{-1}, \nabla(-\Delta+V)^{-1 / 2}$, and $\nabla(-\Delta+V)^{-1} \nabla$. Later, Sugano [2] and Tang-Dong [3] generalized some results of Shen [1] and obtained the estimates for the operators $V^{\beta_{2}}(-\Delta+V)^{-\beta_{1}}, 0 \leq \beta_{2} \leq \beta_{1}<\frac{n}{2}$, and their dual operators. The endpoint properties of Riesz transform and the fractional integral associated to $L$ have been obtained by Yang, Yang, and Zhou [4]. For further information, we refer the reader to Jiang [5], Liu [6], Liu and Dong [7], Sugano [8] and the references therein.

In the study of harmonic analysis and the partial differential equations, the commutators related to singular integral operators play an important role. Let $R_{L}=\nabla(-\Delta+V)^{-1 / 2}$ be the Riesz transform associated with $L$. Based on the results of Shen [1], Guo, Li, and Peng [9] obtained the $L^{p}$-boundedness of the commutators $\left[b, R_{L}\right]$ provided $V \in B_{q}, q \geq n / 2$. By the functional calculus of $L$, Bui [10] obtained the weighted estimate of commutators of some singular integrals related to $L$. We refer to Liu, Huang, and Dong [11], Liu, Wang, and Dong [12], and Wang and Liu [13] for the latest developments on this topic.

In Section 3, assume that $V \in B_{s}, s \geq \frac{n}{2}$. Let $T_{\beta}=(-\Delta+V)^{-\beta} V^{\beta}$ and $b \in \operatorname{BMO}_{\sigma}(\rho)$, where $\operatorname{BMO}_{\sigma}(\rho)$ denotes a function space associated with $L$. Such spaces were first intro-

(c) $2016 \mathrm{Li}$ and Wan. This article is distributed under the terms of the Creative Commons Attribution 4.0 International License (http://creativecommons.org/licenses/by/4.0/), which permits unrestricted use, distribution, and reproduction in any medium, provided you give appropriate credit to the original author(s) and the source, provide a link to the Creative Commons license, and indicate if changes were made. 
duced by Bongioanni, Harboure, and Salinas [14] as a generalization of the bounded mean oscillation space $\operatorname{BMO}\left(\mathbb{R}^{n}\right)$. We investigate the boundedness of the commutator $\left[b, T_{\beta}\right]$ on Herz type spaces $\dot{K}_{q}^{\alpha, p}\left(\mathbb{R}^{n}\right)$ and $K_{q}^{\alpha, p}\left(\mathbb{R}^{n}\right)$, respectively. In Section 3.1, with the help of the $L^{p}$-boundedness of $T_{\beta}$, we verify that the commutators $\left[b, T_{\beta}\right]$ are also bounded on $L^{p}\left(\mathbb{R}^{n}\right)$. See Theorem 3.8. In Section 3.2, we prove that if the index $(\beta, p, q, \alpha)$ satisfies

$$
\left\{\begin{array}{l}
0<\beta<n / 2, \quad 0<p<\infty, \\
s /(s-\beta)<q<\infty, \\
-n / q<\alpha<n(1-1 / q-2 \beta / n),
\end{array}\right.
$$

the operators $T_{\beta}$ and the commutators $\left[b, T_{\beta}\right]$ are bounded on the homogeneous Herz spaces $\dot{K}_{q}^{\alpha, p}\left(\mathbb{R}^{n}\right)$. A similar result also holds for the non-homogeneous Herz spaces $K_{q}^{\alpha, p}\left(\mathbb{R}^{n}\right)$. See Theorems 3.9 and 3.10 for the details.

By use of a maximal estimate, Bongioanni, Harboure, and Salinas [14] proved that the commutators $\left[b, R_{L}\right]$ are bounded on $L^{p}\left(\mathbb{R}^{n}\right)$, where $b \in \operatorname{BMO}_{\sigma}(\rho)$. See Theorem 1 in [14]. In Section 4 , the $L^{p}$-boundedness of $\left[b, R_{L}\right]$ can be further extended to the setting of $\dot{K}_{q}^{\alpha, p}\left(\mathbb{R}^{n}\right)$ and $K_{q}^{\alpha, p}\left(\mathbb{R}^{n}\right)$, respectively. Precisely, we obtain the following results. Suppose that $V \in B_{s}, s \geq \frac{n}{2}$, and $b \in \mathrm{BMO}_{\sigma}(\rho), 0<\sigma<\infty$. Let $\frac{1}{\tilde{q}}=:\left(\frac{1}{s}-\frac{1}{n}\right)^{+}$. If the index $(p, q, \alpha)$ satisfies

$$
\left\{\begin{array}{l}
0<p<\infty,(\tilde{q})^{\prime}<q<\infty, \\
-n / q<\alpha<n(1-1 / q-1 / \tilde{q}),
\end{array}\right.
$$

then $R_{L}$ and $\left[b, R_{L}\right]$ are bounded on $\dot{K}_{q}^{\alpha, p}\left(\mathbb{R}^{n}\right)$ and $K_{q}^{\alpha, p}\left(\mathbb{R}^{n}\right)$. See Theorems 4.4, 4.5 and 4.7.

Remark 1.1 We point out that the results obtained in Sections 3 and 4 cover many former results. It is obvious that

$$
\left\{\begin{array}{l}
\dot{K}_{q}^{0, q}\left(\mathbb{R}^{n}\right)=L^{q}\left(\mathbb{R}^{n}\right), \\
\dot{K}_{q}^{\alpha, q}\left(\mathbb{R}^{n}\right)=L^{q}\left(\mathbb{R}^{n},|x|^{\alpha q}\right) .
\end{array}\right.
$$

Hence our results generalize the $L^{p}$-boundedness of $\left[b, T_{\beta}\right]$ and $\left[b, R_{L}\right]$ to the boundedness of Herz type spaces. On the other hand, Theorems 3.9, 3.10, 4.4 and 4.5 imply that $\left[b, T_{\beta}\right]$ and $\left[b, R_{L}\right]$ are bounded on the Lebesgue spaces $L^{q}\left(\mathbb{R}^{n}\right)$ with power weighted $|x|^{\alpha q}$.

Remark 1.2 For $\beta=1,2, T_{1}=(-\Delta+V)^{-1} V$ and $T_{2}=(-\Delta+V)^{-1 / 2} V^{1 / 2}$. For $b \in \operatorname{BMO}\left(\mathbb{R}^{n}\right)$, the $L^{p}$-boundedness of $\left[b, T_{i}\right], i=1,2$ have been obtained by Guo, Li, and Peng [9], respectively. See Theorem 1 in [9]. In this paper, we assume that $b \in \mathrm{BMO}_{\sigma}(\rho)$, which is larger than $\mathrm{BMO}\left(\mathbb{R}^{n}\right)$. Hence our results cover those of Guo, Li, and Peng [9]. On the other hand, the boundedness of $\left[b, T_{\beta}\right]$ on Herz spaces are new even for the case $b \in \operatorname{BMO}\left(\mathbb{R}^{n}\right)$.

The rest of this paper is organized as follows. In Section 2, we state some notations and known results which will be used throughout this paper. In Section 3, we prove the commutators $\left[b, T_{\beta}\right]$ are bounded on $L^{p}\left(\mathbb{R}^{n}\right)$ and Herz spaces with $b \in \mathrm{BMO}_{\sigma}(\rho)$, respectively. Section 4 is devoted to the boundedness of $\left[b, R_{L}\right]$ on Herz spaces, where $b \in \mathrm{BMO}_{\sigma}(\rho)$. 
Some notations Throughout the paper, the Lebesgue measure of the ball $B \subset \mathbb{R}^{n}$ denote by $|B| . c$ and $C$ will denote unspecified positive constants, possibly different at each occurrence. The constants are independent of the functions. $\mathrm{U} \approx \mathrm{V}$ represents that there is a constant $c>0$ such that $c^{-1} \mathrm{~V} \leq \mathrm{U} \leq c \mathrm{~V}$ whose right inequality is also written as $\mathrm{U} \lesssim \mathrm{V}$. Similarly, if $\mathrm{V} \geq c \mathrm{U}$, we denote $\mathrm{V} \gtrsim \mathrm{U}$.

\section{Preliminaries}

\subsection{Auxiliary function and BMO type spaces}

In this paper, we consider the Schrödinger differential operator $L=-\Delta+V$ on $\mathbb{R}^{n}, n \geq 3$, where $V$ is a nonnegative potential belonging to the reverse Hölder class $B_{s}, s \geq \frac{n}{2}$, which is defined as follows.

Definition 2.1 A nonnegative locally $L^{s}$-integrable function $V$ on $\mathbb{R}^{n}$ is said to belong to $B_{s}, s>1$, if there exists $C>0$ such that the reverse Hölder inequality

$$
\left(\frac{1}{|B|} \int_{B} V^{s}(x) d x\right)^{\frac{1}{s}} \lesssim\left(\frac{1}{|B|} \int_{B} V(x) d x\right)
$$

holds for every ball $B \subset \mathbb{R}^{n}$.

Remark 2.2 Assume that $V \in B_{s}, 1<s<\infty$. Then $V(y) d y$ is a doubling measure. Namely, there exists a constant $C_{0}$ such that for any $r>0$ and $y \in \mathbb{R}^{n}$,

$$
\int_{B(x, 2 r)} V(y) d y \lesssim C_{0} \int_{B(x, r)} V(y) d y
$$

Lemma 2.3 ([1], Lemma 1.2) Suppose that $V \in B_{s}, s>\frac{n}{2}$. There exists a constant $C$ such that for $0<r<r_{0}<\infty$,

$$
\frac{1}{r^{n-2}} \int_{B(x, r)} V(y) d y \lesssim\left(\frac{r_{0}}{r}\right)^{n / s-2} \cdot \frac{1}{r_{0}^{n-2}} \int_{B\left(x, r_{0}\right)} V(y) d y
$$

In order to study the Neumann problem for the Schrödinger operator, Shen [15] introduced the following auxiliary function.

Definition 2.4 For $x \in \mathbb{R}^{n}$, the function $\rho$ is defined by

$$
\rho(x)=\sup \left\{r>0: \frac{1}{r^{n-2}} \int_{B(x, r)} V(y) d y \leq 1\right\} .
$$

We list some properties of the auxiliary function $\rho$ which will be used in the sequel.

Lemma 2.5 ([1], Lemma 1.4) There exist positive constants $C>0, c>0$, and $l_{0}>0$ such that, for $x, y$ in $\mathbb{R}^{n}$,
(a) $\rho(x) \sim \rho(y)$ if $|x-y| \leq \frac{C}{\rho(x)}$,
(b) $\rho(x) \leq C(1+|x-y| / \rho(x))^{l_{0}} \rho(y)$,
(c) $\rho(x)\{1+|x-y| / \rho(x)\}^{l_{0} /\left(l_{0}+1\right)} \geq c \rho(y)$. 
Lemma 2.6 ([9], Lemma 2.3) Suppose $V \in B_{s}, s>\frac{n}{2}$. Then for any $N_{0}>\log _{2} C_{0}+1$, where $C_{0}$ is the constant in (2.1), there exists a constant $C_{N_{0}}$ such that for any $x \in \mathbb{R}^{n}$ and $r>0$,

$$
\frac{1}{(1+r / \rho(x))^{N_{0}}} \int_{B(x, r)} V(y) d y \lesssim C_{N_{0}} r^{n-2}
$$

The bounded mean oscillation space $\mathrm{BMO}\left(\mathbb{R}^{n}\right)$ was first introduced by John-Nirenberg [16] to study some problems arising from elasticity theory. In harmonic analysis, it is well known that $\operatorname{BMO}\left(\mathbb{R}^{n}\right)$ is the dual of Hardy space $H^{1}\left(\mathbb{R}^{n}\right)$. In [14], Bongioanni, Harboure, and Salinas added a perturbation term into the definition of $\mathrm{BMO}\left(\mathbb{R}^{n}\right)$ and introduced a new class of BMO type spaces associated with Schrödinger operators. See also [17, 18]. Given a function $b \in L_{\mathrm{loc}}^{1}$ and a cube $B$, let $b_{B}$ denote the average of $b$ on $B$ :

$$
b_{B}=\frac{1}{|B|} \int_{B} b(x) d x
$$

Definition 2.7 Suppose $V \in B_{s}, s>n / 2$, and $\sigma \in(0, \infty)$.

(i) A new space $\operatorname{BMO}_{\sigma}(\rho)$ is defined as the set of all $b \in L_{\text {loc }}^{1}$ which satisfies

$$
\frac{1}{|B(x, r)|} \int_{B(x, r)}\left|b(y)-b_{B}\right| d y<C\left(1+\frac{r}{\rho(x)}\right)^{\sigma}
$$

where the supremum is taken over all cubes $B(x, r) \in \mathbb{R}^{n}$. A norm for $b \in \operatorname{BMO}_{\sigma}(\rho)$, denoted by $\|b\|_{\sigma}$ is given by the infimum of the constants satisfying (2.2), after identifying functions that differ upon a constant.

(ii) With the above definition in mind, we define $\mathrm{BMO}_{\infty}(\rho)=\bigcup_{\sigma>0} \mathrm{BMO}_{\sigma}(\rho)$.

Proposition $2.8([14])$ For $0<\sigma<\sigma^{\prime}, \mathrm{BMO}\left(\mathbb{R}^{n}\right) \subset \mathrm{BMO}_{\sigma}(\rho) \subset \mathrm{BMO}_{\sigma^{\prime}}(\rho)$.

Bongioanni, Harboure, and Salinas [14] gave some examples to clarify that $\mathrm{BMO}\left(\mathbb{R}^{n}\right)$ is a subspace of $\mathrm{BMO}_{\infty}(\rho)$.

Lemma 2.9 ([14], Proposition 3) Let $\sigma>0$ and $1<p<\infty$. If $b \in \mathrm{BMO}_{\sigma}(\rho)$, then for all $B=B(x, r)$ with $x \in \mathbb{R}^{n}$ and $r>0$,

$$
\left(\frac{1}{|B|} \int_{B}\left|b(y)-b_{B}\right|^{p} d y\right)^{\frac{1}{p}} \leq\|b\|_{\sigma}\left(1+\frac{r}{\rho(x)}\right)^{\sigma^{\prime}}
$$

where $\sigma^{\prime}=\left(l_{0}+1\right) \sigma$ and $l_{0}$ is the constant appearing in Lemma 2.5 .

The following result can be deduced from Lemma 2.9 immediately.

Lemma 2.10 ([14], Lemma 1) Let $\sigma>0$ and $1<p<\infty$. If $b \in \mathrm{BMO}_{\sigma}(\rho), B=B(x, r)$, and $B_{B_{k}}=B\left(x, 2^{k} r\right)$, then for $x \in \mathbb{R}^{n}$ and $r>0$ with $\sigma^{\prime}=\left(l_{0}+1\right) \sigma$,

$$
\left(\frac{1}{\left|B_{B_{k}}\right|} \int_{B_{B_{k}}}\left|b(y)-b_{B}\right|^{p} d y\right)^{\frac{1}{p}} \leq\|b\|_{\sigma} k\left(1+\frac{2^{k} r}{\rho(x)}\right)^{\sigma^{\prime}} .
$$




\subsection{Herz spaces}

We state some basic knowledge on the Herz spaces. In 1968, in order to study absolutely convergent Fourier transforms, Herz [19] introduced a new class of function spaces $K_{q}$ which are equivalent to the homogeneous Herz spaces with special exponent. In 1990s, Lu and Yang introduced the homogeneous Herz spaces and non-homogeneous Herz spaces with general indices. By the block decomposition, Lu and Yang obtained many interesting properties of these spaces. We refer the reader to $\mathrm{Lu}$, Yang, and $\mathrm{Hu}$ [20] for the details [21-24] and the references therein.

For $k \in \mathbb{Z}$, let $B_{k}=\left\{x \in \mathbb{R}^{n}:|x| \leq 2^{k}\right\}$ and $E_{k}=B_{k} \backslash B_{k-1}$. Denote by $\chi_{k}$ the characteristic function of $E_{k}$. Define

$$
\tilde{\chi}_{k}= \begin{cases}\chi_{k}, & k \in \mathbb{N}, \\ \chi_{B_{0}}, & k=0 .\end{cases}
$$

The Herz spaces are defined as follows.

Definition 2.11 Let $\alpha \in \mathbb{R}, 0<p, q \leq \infty$.

(i) The homogeneous Herz space $\dot{K}_{q}^{\alpha, p}\left(\mathbb{R}^{n}\right)$ is defined by

$$
\dot{K}_{q}^{\alpha, p}\left(\mathbb{R}^{n}\right)=\left\{f \in L_{\mathrm{loc}}^{q}\left(\mathbb{R}^{n} \backslash 0\right):\|f\|_{\dot{K}_{q}^{\alpha, p}\left(\mathbb{R}^{n}\right)}<\infty\right\},
$$

where

$$
\|f\|_{K_{q}^{\alpha, p}\left(\mathbb{R}^{n)}\right.}=\left\{\sum_{k \in \mathbb{Z}} 2^{k \alpha p}\left\|f \chi_{k}\right\|_{L^{q}\left(\mathbb{R}^{n)}\right.}\right\}^{\frac{1}{q}} .
$$

(ii) The non-homogeneous Herz space $K_{q}^{\alpha, p}\left(\mathbb{R}^{n}\right)$ is defined by

$$
K_{q}^{\alpha, p}\left(\mathbb{R}^{n}\right)=\left\{f \in L_{\mathrm{loc}}^{q}\left(\mathbb{R}^{n}\right):\|f\|_{K_{q}^{\alpha, p}\left(\mathbb{R}^{n}\right)}<\infty\right\},
$$

where

$$
\|f\|_{K_{q}^{\alpha, p}\left(\mathbb{R}^{n)}\right.}=\left\{\sum_{k=0}^{\infty} 2^{k \alpha p}\left\|f \widetilde{\chi}_{k}\right\|_{L^{q}\left(\mathbb{R}^{n)}\right.}\right\}^{\frac{1}{q}} .
$$

Proposition 2.12 ([20], Proposition 1.1.2) Let $0<p, q \leq \infty$ and $0<\alpha<\infty$. Then

$$
K_{q}^{\alpha, p}\left(\mathbb{R}^{n}\right)=\dot{K}_{q}^{\alpha, p}\left(\mathbb{R}^{n}\right) \cap L^{q}\left(\mathbb{R}^{n}\right) .
$$

Forf $\in K_{q}^{\alpha, p}\left(\mathbb{R}^{n}\right)$,

$$
\|f\|_{K_{q}^{\alpha, p}\left(\mathbb{R}^{n}\right)} \approx\|f\|_{\dot{K}_{q}^{\alpha, p}\left(\mathbb{R}^{n}\right)}+\|f\|_{L^{q}\left(\mathbb{R}^{n}\right)} .
$$

Let $X$ be a function space. We denote by $X^{*}$ the dual of $X$. For the homogeneous and non-homogeneous Herz spaces, there exist the following dual relations, respectively.

Lemma 2.13 ([20], Corollary 1.2.1) Let $\alpha \in \mathbb{R}, 0<p<\infty$, and $1 \leq q<\infty$. Then:

(i) $\left(\dot{K}_{q}^{\alpha, p}\left(\mathbb{R}^{n}\right)\right)^{*}=\dot{K}_{q^{\prime}}^{-\alpha, p^{\prime}}\left(\mathbb{R}^{n}\right)$.

(ii) $\left(K_{q}^{\alpha, p}\left(\mathbb{R}^{n}\right)\right)^{*}=K_{q^{\prime}}^{-\alpha, p^{\prime}}\left(\mathbb{R}^{n}\right)$. 


\subsection{Some lemmas}

Let $L=-\Delta+V$ be the Schrödinger operator with $V \in B_{s}, s \geq \frac{n}{2}$. The heat semigroup associated with $L$ is defined as

$$
T_{t} f(x)=e^{-t L} f(x)=\int_{\mathbb{R}^{n}} K_{t}(x, y) f(y) d y, \quad f \in L^{2}\left(\mathbb{R}^{n}\right), t>0 .
$$

Lemma 2.14 ([25], Theorem 4.10) Let $K_{t}(x, y)$ be the kernel defined in (2.3). For every nonnegative integer $N$, there is a constant $C_{N}$ such that

$$
0 \leq K_{t}(x, y) \lesssim C_{N} t^{-\frac{n}{2}} \exp \left(-\frac{|x-y|^{2}}{5 t}\right)(1+\sqrt{t} / \rho(x)+\sqrt{t} / \rho(y))^{-N}
$$

For $0<\beta<\frac{n}{2}$, the fractional integral associated with $L$ is defined by

$$
L^{-\beta} f(x)=\int_{0}^{\infty} e^{-t L} f(x) t^{\beta-1} d t
$$

The following result is obtained by Tang and Dong [3].

Lemma 2.15 ([3]) Suppose $V \in B_{s}, s \geq \frac{n}{2}$. For every $N \in \mathbb{N}$, there exists a constant $C_{N}$ such that

$$
\int_{0}^{\infty} t^{\beta-1} K_{t}(x, y) d t \leq \frac{C_{N}}{(1+|x-y| / \rho(x))^{N}} \frac{1}{|x-y|^{n-2 \beta}} .
$$

Denote by $K_{\beta}(\cdot, \cdot)$ the kernel of the operator $T_{\beta}=(-\Delta+V)^{-\beta} V^{\beta}$. The following pointwise estimate of $K_{\beta}$ is a direct corollary of Lemma 2.15. We omit the details.

Lemma 2.16 Suppose that $V \in B_{s}, s \geq \frac{n}{2}$. For every $N \in \mathbb{N}$, there exist constants $C_{N}$ and $\delta>0$ such that for $0<h<\frac{|x-y|}{16}$,

$$
\left|K_{\beta}(x, y)\right| \leq \frac{C_{N}}{(1+|x-y| / \rho(x))^{N}} \frac{1}{|x-y|^{n-2 \beta}} V^{\beta}(y)
$$

and

$$
\left|K_{\beta}(x+h, y)-K_{\beta}(x, y)\right| \leq \frac{C_{N}}{(1+|x-y| / \rho(x))^{N}} \frac{|h|^{\delta}}{|x-y|^{n-2 \beta+\delta}} V^{\beta}(y) .
$$

For the sake of convenience, in the sequel, we take $N$ large enough such that $N /\left(l_{0}+1\right)-$ $\sigma\left(l_{0}+1\right)-N_{0} \beta>0$, where $N_{0}$ is the constant in Lemma 2.6.

Lemma 2.17 Suppose that $V \in B_{s}$ with $s \geq \frac{n}{2}$. Let $x \in E_{k}, y \in E_{j}$.

(a) If $j \leq k-2$, then $\frac{1}{(1+|x-y| \mid \rho(x))^{N}} \leq \frac{1}{\left(1+2^{k} / \rho(0)\right)^{N /\left(l_{0}+1\right)}}$.

(b) If $j \geq k+2$, then $\frac{1}{(1+|x-y| / \rho(x))^{N}} \leq \frac{1}{(1+2 / \rho(0))^{N /\left(l_{0}+1\right)}}$. 
Proof We only prove (a), and (b) can be dealt with similarly. For $x \in E_{k}$ and $y \in E_{j}$ with $j \leq k-2$, by a simple computation, we can see that $|x-y| \sim 2^{k}$. By (c) of Lemma 2.5 , we have

$$
\begin{aligned}
\frac{1}{(1+|x-y| / \rho(x))^{N}} & \leq\left(\frac{1}{1+|x-y| \frac{1}{\rho(0)(1+|x| / \rho(0))_{0}^{l_{0} / l_{0}+1}}}\right)^{N} \\
& \leq\left(\frac{(1+|x| / \rho(0))^{l_{0} / l_{0}+1}}{(1+|x| / \rho(0))^{l_{0} / l_{0}+1}+|x-y| / \rho(0)}\right)^{N} \\
& \leq\left(\frac{(1+|x| / \rho(0))^{l_{0} / l_{0}+1}}{1+|x-y| / \rho(0)}\right)^{N} \\
& \leq \frac{1}{\left(1+2^{k} / \rho(0)\right)^{N /\left(l_{0}+1\right)}} .
\end{aligned}
$$

A ball centered at $x$ and with radius $\rho(x)$ is called critical. In this paper, we use the symbol $B(x, \rho(x))$ to denote the critical ball. Dziubański and Zienkiewicz [26] gave the following covering lemma.

Lemma 2.18 ([26], Lemma 2.3) There exists a sequence of points $\left\{x_{k}\right\}_{k=1}^{\infty} \subset \mathbb{R}^{n}$, such that the family of critical balls $\left\{Q_{k}=B\left(x_{k}, \rho\left(x_{k}\right)\right)\right\}_{k>1}^{\infty}$ satisfies:

(i) $\mathbb{R}^{n}=\bigcup_{k} Q_{k}$.

(ii) There exists $N=N(\rho)$ such that for every $k \in N$,

$$
\operatorname{card}\left\{j: 4 Q_{j} \cap 4 Q_{k}\right\} \leq N
$$

Definition 2.19 Let $\gamma>0$ and $\mathfrak{B}_{\rho, \gamma}$ be the set $\left\{B(y, r): y \in \mathbb{R}^{n}, r \leq \gamma \rho(y)\right\}$. For $f \in L_{\mathrm{loc}}^{1}\left(\mathbb{R}^{n}\right)$ and $x \in \mathbb{R}^{n}$, we define the following two maximal functions:

$$
M_{\rho, \gamma}(f)(x)=\sup _{x \in B \in \mathfrak{B}_{\rho, \gamma}} \frac{1}{|B|} \int_{B}|f(y)| d y
$$

and

$$
M_{\rho, \gamma}^{\sharp}(f)(x)=\sup _{x \in B \in \mathfrak{B}_{\rho, \gamma}} \frac{1}{|B|} \int_{B}\left|f(y)-f_{B}\right| d y .
$$

Definition 2.20 Let $\mathfrak{F}_{Q}$ be the set $\left\{B(y, r): y \in \mathbb{R}^{n}, r>0\right\}$ and let $Q$ be a ball in $\mathbb{R}^{n}$. For $f \in L_{\text {loc }}^{1}\left(\mathbb{R}^{n}\right)$ and $y \in Q$, we define

$$
M_{Q}(f)(x)=\sup _{x \in B \in \mathfrak{F}_{Q}} \frac{1}{|B \cap Q|} \int_{B \cap Q}|f(y)| d y
$$

and

$$
M_{Q}^{\sharp}(f)(x)=\sup _{x \in B \in \mathfrak{F}_{Q}} \frac{1}{|B \cap Q|} \int_{B \cap Q}\left|f(y)-f_{B \cap Q}\right| d y .
$$

In the proof of Theorem 3.8 below, we need the following Fefferman-Stein type inequality. 
Lemma 2.21 ([14], Lemma 2) For $1<p<\infty$, there exist $\xi$ and $\gamma$ such that if $\left\{Q_{k}\right\}_{k=1}^{\infty}$ is a sequence of the balls as those in Lemma 2.18, then for all $f \in L_{\mathrm{loc}}^{1}\left(\mathbb{R}^{n}\right)$,

$$
\int_{\mathbb{R}^{n}}\left|M_{\rho, \xi}(f)(x)\right|^{p} d x \lesssim \int_{\mathbb{R}^{n}}\left|M_{\rho, \gamma}^{\sharp}(f)(x)\right|^{p} d x+\sum_{k}\left|Q_{k}\right|\left(\frac{1}{|Q|} \int_{2 Q_{k}}|f(x)| d x\right)^{p} .
$$

\section{Commutators $\left[b, T_{\beta}\right]$ on Herz type spaces}

\subsection{The boundedness of $\left[b, T_{\beta}\right]$ on $L^{p}\left(\mathbb{R}^{n}\right)$}

Throughout this paper, for $p \in(1, \infty)$, denote by $p^{\prime}$ the conjugate of $p$, that is, $\frac{1}{p}+\frac{1}{p^{\prime}}=1$. As usual, for $f \in L_{\text {loc }}^{1}\left(\mathbb{R}^{n}\right)$, the $p$-maximal function $M_{p}$ is defined as

$$
M_{p} f(x)=\sup _{r>0}\left(\frac{1}{|B(x, r)|} \int_{B(x, r)}|f(y)|^{p} d y\right)^{\frac{1}{p}} .
$$

Definition 3.1 Let $f \in L_{\mathrm{loc}}^{q}\left(\mathbb{R}^{n}\right)$. The fractional Hardy-Littlewood maximal operator $M_{\sigma, \gamma}$ is defined by

$$
M_{\sigma, \gamma} f(x)=\sup _{x \in B}\left(\frac{1}{|B|^{1-\frac{\sigma \gamma}{n}}} \int_{B}|f(y)|^{\gamma} d y\right)^{\frac{1}{\gamma}} .
$$

Lemma 3.2 ([27]) Suppose that $1<\gamma<p_{1}<\frac{n}{\sigma}$ and $\frac{1}{p_{2}}=\frac{1}{p_{1}}-\frac{\sigma}{n}$. Then

$$
\left\|M_{\sigma, \gamma} f\right\|_{L^{p_{2}\left(\mathbb{R}^{n}\right)}} \lesssim\|f\|_{L^{p_{1}\left(\mathbb{R}^{n}\right)} .}
$$

Now we consider the boundedness of $T_{\beta}=(-\Delta+V)^{-\beta} V^{\beta}, \beta>0$. The following maximal estimate is obtained by Li, Wan, and Zhang [28].

Lemma 3.3 ([28], Theorem 4.8) Suppose that $V \in B_{s}$ for $s>\frac{n}{2}$. Let $0<\beta_{2} \leq \beta_{1}<\frac{n}{2}, 1<$ $\left(\frac{s}{\beta_{2}}\right)^{\prime}<p_{1}<\frac{n}{2\left(\beta_{1}-\beta_{2}\right)}$ and $\frac{1}{p_{2}}=\frac{1}{p_{1}}-\frac{2 \beta_{1}-2 \beta_{2}}{n}$. Then

$$
\left|(-\Delta+V)^{-\beta_{1}} V^{\beta_{2}} f(x)\right| \lesssim M_{2\left(\beta_{1}-\beta_{2}\right),\left(\frac{s}{\beta_{2}}\right)^{\prime}} f(x) .
$$

Let $\beta_{1}=\beta_{2}$. By Lemma 3.3, we can obtain the following results.

Corollary 3.4 Suppose that $V \in B_{s}, s \geq \frac{n}{2}$. Let $1<\beta<\frac{n}{2}$.

(i) For $\frac{s}{s-\beta}<p<\infty, T_{\beta}$ is bounded on $L^{p}$.

(ii) For $1<p<\frac{s}{\beta}, T_{\beta}^{*}$ is bounded on $L^{p}$.

Now, let $b \in \mathrm{BMO}_{\sigma}(\rho)$. We consider the $L^{p}$-boundedness of the commutator $\left[b, T_{\beta}\right]$. We need the following maximal function estimate of $\left[b, T_{\beta}\right]$.

Theorem 3.5 Suppose that $V \in B_{s}, s \geq \frac{n}{2}$, and $b \in \mathrm{BMO}_{\sigma}(\rho), 0<\sigma<\infty$. If $\frac{s}{s-\beta}<p<\infty$, there exists a constant $C>0$ such that, for all $f \in L_{\mathrm{loc}}^{p}\left(\mathbb{R}^{n}\right)$ and every critical ball $Q=$ $B\left(x_{0}, \rho\left(x_{0}\right)\right)$,

$$
\frac{1}{|Q|} \int_{Q}\left|\left[b, T_{\beta}\right] f(x)\right| d x \leq C\|b\|_{\sigma}\left\{\inf _{x \in Q} M_{p} f(x)+\inf _{x \in Q} M_{p}\left(T_{\beta} f\right)(x)\right\} .
$$


Proof For any constant $a, b(x)-b(y)=(b(x)-a)-(b(y)-a)$. Then we have

$$
\begin{aligned}
{\left[b, T_{\beta}\right] f(x) } & =\int_{\mathbb{R}^{n}}(b(x)-b(y)) K_{\beta}(x, y) f(y) d y \\
& =(b(x)-a) \int_{\mathbb{R}^{n}} K_{\beta}(x, y) f(y) d y+T_{\beta}((b-a) f)(x) \\
& =I_{1}+I_{2} .
\end{aligned}
$$

Let $f \in L^{p}\left(\mathbb{R}^{n}\right)$ and $Q=B\left(x_{0}, \rho\left(x_{0}\right)\right)$ with $a=b_{2 Q}$. We deal with the average of $I_{1}$ and $I_{2}$ on $Q$, respectively. At first, by Lemma 2.9 and Hölder's inequality with $p>\frac{s}{s-\beta}$, we can get

$$
\begin{aligned}
\frac{1}{|Q|} \int_{Q}\left|I_{1}\right| d x & \lesssim \frac{1}{|Q|} \int_{Q}\left|b(x)-b_{2 Q}\right|\left|T_{\beta} f(x)\right| d x \\
& \lesssim\left\{\frac{1}{|Q|} \int_{Q}\left|b(x)-b_{2 Q}\right|^{p^{\prime}} d x\right\}^{\frac{1}{p^{\prime}}}\left\{\frac{1}{|Q|} \int_{Q}\left|T_{\beta} f(x)\right|^{p} d x\right\}^{\frac{1}{p}} \\
& \lesssim\|b\|_{\sigma} \inf _{x \in Q} M_{p}\left(T_{\beta} f\right)(x) .
\end{aligned}
$$

For $I_{2}$, we split $f=f_{1}+f_{2}$, where $f_{1}(x)=f(x) \chi_{2 Q}(x)$ and $f_{2}(x)=f(x) \chi_{(2 Q) c}(x)$. Take $p_{0} \in\left(\frac{s}{s-\beta}, p\right)$ and denote $\mu=\frac{p}{p-p_{0}}$. By Corollary 3.4 and Lemma 2.9, we apply Hölder's inequality to get

$$
\begin{aligned}
\frac{1}{|Q|} \int_{Q}\left|T_{\beta}\left(\left(b-b_{2 Q}\right) f_{1}\right)(x)\right| d x \\
\quad \lesssim\left(\frac{1}{|Q|} \int_{Q}\left|T_{\beta}\left(\left(b-b_{2 Q}\right) f_{1}\right)(x)\right|^{p_{0}} d x\right)^{\frac{1}{p_{0}}} \\
\quad \lesssim\left\{\frac{1}{|Q|} \int_{2 Q}\left|\left(b(x)-b_{2 Q}\right) f(x)\right|^{p_{0}} d x\right\}^{\frac{1}{p_{0}}} \\
\quad \lesssim\left\{\frac{1}{|Q|} \int_{2 Q}|f(x)|^{p} d x\right\}^{\frac{1}{p}}\left\{\frac{1}{|Q|} \int_{2 Q}^{\left.\left|b(x)-b_{2 Q}\right|^{\mu p_{0}} d x\right\}^{\frac{1}{\mu p_{0}}}}\right. \\
\quad \lesssim\|b\|_{\sigma} \inf _{x \in Q} M_{p}(f)(x) .
\end{aligned}
$$

Next we deal with the term

$$
\frac{1}{|Q|} \int_{Q}\left|T_{\beta}\left(\left(b-b_{2 Q}\right) f_{2}\right)(x)\right| d x .
$$

If $x \in B\left(x_{0}, \rho\left(x_{0}\right)\right)$ and $z \in B\left(x_{0}, 2^{j} \rho\left(x_{0}\right)\right)$ with $j \geq 2$, then $|x-z| \sim\left|x_{0}-z\right|$. With the help of Lemmas 2.17 and 2.10, we use Hölder's inequality to deduce that

$$
\begin{aligned}
& T_{\beta}\left(\left(b-b_{2 Q}\right) f_{2}\right)(x) \\
& \quad \lesssim \int_{\left|x_{0}-z\right|>2 \rho\left(x_{0}\right)} \frac{\left|b(z)-b_{2 Q}\right||f(z)| V^{\beta}(z)}{\left(1+\frac{\left|x_{0}-z\right|}{\rho\left(x_{0}\right)}\right)^{N_{1}}\left|x_{0}-z\right|^{n-2 \beta}} d z \\
& \quad \lesssim \sum_{j=1}^{\infty}\left(1+2^{j}\right)^{-N_{1}} \frac{1}{\left|2^{j} \rho\left(x_{0}\right)\right|^{n-2 \beta}} \int_{2^{j} \rho\left(x_{0}\right)<\left|x_{0}-z\right|<2^{j+1} \rho\left(x_{0}\right)}\left|b(z)-b_{2 Q}\right||f(z)| V^{\beta}(z) d z
\end{aligned}
$$




$$
\begin{aligned}
& \lesssim \sum_{j=1}^{\infty}\left(1+2^{j}\right)^{-N_{1}} \frac{\left|2^{j+1} \rho\left(x_{0}\right)\right|^{n}}{\left|2^{j} \rho\left(x_{0}\right)\right|^{n-2 \beta}}\left\{\frac{1}{\left|Q_{2^{j+1}}\right|} \int_{Q_{2^{j+1}}}\left|b(z)-b_{2 Q}\right|^{p_{1}} d z\right\}^{\frac{1}{p_{1}}} \\
& \quad \times\left\{\frac{1}{\left|Q_{2^{j+1}}\right|} \int_{Q_{2^{j+1}}}|f(z)|^{p} d z\right\}^{\frac{1}{p}}\left\{\frac{1}{\left|Q_{2^{j+1}}\right|} \int_{Q_{2^{j+1}}}|V(z)|^{s} d z\right\}^{\frac{\beta}{s}} \\
& \lesssim \sum_{j=1}^{\infty} j\left(1+2^{j}\right)^{-N_{2}} \frac{\left|2^{j} \rho\left(x_{0}\right)\right|^{(n-2) \beta}}{\left|2^{j} \rho\left(x_{0}\right)\right|^{-2 \beta}\left|Q_{2^{j}}\right|^{\beta}}\|b\|_{\sigma} \inf _{y \in Q} M_{p}(f)(y) \\
& \lesssim\|b\|_{\sigma} \inf _{y \in Q} M_{p}(f)(y)
\end{aligned}
$$

where $Q_{2^{j+1}}=B\left(x_{0}, 2^{j+1} \rho\left(x_{0}\right)\right), \frac{\beta}{s}+\frac{1}{p}+\frac{1}{p_{1}}=1$, and $N_{1}=N /\left(l_{0}+1\right)$ and $N_{2}=N /\left(l_{0}+1\right)-\sigma\left(l_{0}+\right.$ 1) $-N_{0} \beta$.

Finally, in Lemma 2.15, take $N$ large enough such that $N>N_{2}$. We complete the proof of Theorem 3.5.

Remark 3.6 It is easy to check that if the critical ball $Q$ is replaced by $2 Q$, Theorem 3.5 also holds.

Lemma 3.7 Let $V \in B_{s}, s \geq \frac{n}{2}$, and $b \in \mathrm{BMO}_{\sigma}(\rho), 0<\sigma<\infty$. Then for any $p>\frac{s}{s-\beta}$ and $\gamma \geq 1$, there exists a constant $C$ such that for all $f$ and $x, y \in B=B\left(x_{0}, r\right)$ with $r<\gamma \rho\left(x_{0}\right)$,

$$
\int_{(2 B)^{c}}|K(x, z)-K(y, z)| V^{\beta}(z)\left|b(z)-b_{B}\right||f(z)| d z \leq C\|b\|_{\sigma} \inf _{u \in B} M_{p} f(u) .
$$

Proof Write $E_{j}=B\left(x_{0}, 2^{j+1} r\right) \backslash B\left(x_{0}, 2^{j} r\right)$. For $x \in B\left(x_{0}, r\right)$ and $z \in E_{j}$, we have $|x-z| \sim\left|x_{0}-z\right|$. With the help of Lemmas 2.17, 2.10 and 2.6, we obtain

$$
\begin{aligned}
& \int_{(2 B)^{c}}|K(x, z)-K(y, z)| V^{\beta}(z)\left|b(z)-b_{B}\right||f(z)| d z \\
& \quad \lesssim \sum_{j=1}^{\infty} \int_{E_{j}} \frac{\left|b(z)-b_{B}\right||f(z)| V^{\beta}(z)|r|^{\delta}}{\left(1+\frac{\left|x-z_{0}\right|}{\rho\left(x_{0}\right)}\right)^{N_{1}}\left|x-z_{0}\right|^{n-2 \beta+\delta}} d z \\
& \lesssim \sum_{j=1}^{\infty} \frac{1}{\left(1+\frac{2^{j} r}{\rho\left(x_{0}\right)}\right)^{N_{1}}} \frac{|r|^{\delta}}{\left(2^{j} r\right)^{n-2 \beta+\delta}} \int_{E_{j}}\left|b(z)-b_{B}\right||f(z)| V^{\beta}(z) d z \\
& \lesssim \sum_{j=1}^{\infty} \frac{j 2^{-j \delta}}{\left(1+\frac{2^{j} r}{\rho\left(x_{0}\right)}\right)^{N_{2}}}\|b\|_{\sigma} \inf _{z \in Q} M_{p}(f)(z) \\
& \lesssim\|b\|_{\sigma} \inf _{z \in Q} M_{p}(f)(z),
\end{aligned}
$$

where $N_{1}=N /\left(l_{0}+1\right)$ and $N_{2}=N /\left(l_{0}+1\right)-\sigma\left(l_{0}+1\right)-N_{0} \beta$. Taking $N$ large enough, we complete the proof of Lemma 3.7.

With the help of Lemma 3.7, we can obtain the $L^{p}$-boundedness of $\left[b, T_{\beta}\right]$. Denote by $T_{\beta}^{\prime}$ the dual operator of $T_{\beta}$. We have the following.

Theorem 3.8 Let $V \in B_{s}, s \geq \frac{n}{2}$, and let $b \in \mathrm{BMO}_{\sigma}(\rho), 0<\sigma<\infty$. 
(i) If $1<\beta<\frac{n}{2}, \frac{s}{s-\beta}<p<\infty$, then

$$
\left\|\left[b, T_{\beta}\right] f\right\|_{L^{p}\left(\mathbb{R}^{n}\right)} \lesssim\|b\|_{\sigma}\|f\|_{L^{p}\left(\mathbb{R}^{n}\right)} .
$$

(ii) If $1<\beta<\frac{n}{2}, 1<p<\frac{s}{\beta}$, then

$$
\left\|\left[b, T_{\beta}^{*}\right] f\right\|_{L^{p}\left(\mathbb{R}^{n)}\right.} \lesssim\|b\|_{\sigma}\|f\|_{L^{p}\left(\mathbb{R}^{n}\right)}
$$

Proof We only prove (i), and (ii) follows by duality. For $f \in L^{p}\left(\mathbb{R}^{n}\right), \frac{s}{s-\beta}<p<\infty$, by Theorem 3.5, we can see that $\left[b, T_{\beta}\right] f \in L_{\text {loc }}^{1}\left(\mathbb{R}^{n}\right)$. By Theorem 3.5, Lemma 3.7, and Remark 3.6, we have

$$
\begin{aligned}
\left\|\left[b, T_{\beta}\right] f\right\|_{L^{q}\left(\mathbb{R}^{n}\right)}^{q} \lesssim & \int_{\mathbb{R}^{n}}\left|M_{\rho, \xi}\left(\left[b, T_{\beta}\right] f\right)(x)\right|^{q} d x \\
\lesssim & \int_{\mathbb{R}^{n}}\left|M_{\rho, \gamma}^{\sharp}\left(\left[b, T_{\beta}\right] f\right)(x)\right|^{q} d x+\sum_{k}\left|Q_{k}\right|\left(\frac{1}{\left|Q_{k}\right|} \int_{2 Q_{k}}\left|\left[b, T_{\beta}\right] f(x)\right| d x\right)^{q} \\
\lesssim & \int_{\mathbb{R}^{n}}\left|M_{\rho, \gamma}^{\sharp}\left(\left[b, T_{\beta}\right] f\right)(x)\right|^{q} d x \\
& +\|b\|_{\sigma}\left(\sum_{k} \int_{2 Q_{k}}\left|M_{p} f(x)\right|^{q} d x+\sum_{k} \int_{2 Q_{k}}\left|M_{p}\left(T_{\beta} f\right)(x)\right|^{q} d x\right) \\
\lesssim & \int_{\mathbb{R}^{n}}\left|M_{\rho, \gamma}^{\sharp}\left(\left[b, T_{\beta}\right] f\right)(x)\right|^{q} d x+\|b\|_{\sigma}\left(\|f\|_{L^{q}\left(\mathbb{R}^{n)}\right.}^{q}+\left\|T_{\beta} f\right\|_{L^{q}\left(\mathbb{R}^{n}\right)}^{q}\right) \\
\lesssim & \int_{\mathbb{R}^{n}}\left|M_{\rho, \gamma}^{\sharp}\left(\left[b, T_{\beta}\right] f\right)(x)\right|^{q} d x+\|b\|_{\sigma}\|f\|_{L^{q}\left(\mathbb{R}^{n}\right)}^{q},
\end{aligned}
$$

where the last but one inequality is due to Lemma 2.18, and the boundedness of $M_{p}$ in $L^{q}\left(\mathbb{R}^{n}\right)$ for $p<q$. Next, we consider the term

$$
\int_{\mathbb{R}^{n}}\left|M_{\rho, \gamma}^{\sharp}\left(\left[b, T_{\beta}\right] f\right)(x)\right|^{q} d x .
$$

We have $\left[b, T_{\beta}\right] f(x)=: B_{1}(x)+B_{2}(x)$, where

$$
\left\{\begin{array}{l}
B_{1}(x)=:\left(b(x)-b_{B}\right) T_{\beta} f(x), \\
B_{2}(x)=: T_{\beta}\left(\left(b-b_{B}\right) f\right)(x) .
\end{array}\right.
$$

This gives

$$
\begin{aligned}
& \frac{1}{|B|} \int_{B}\left|\left[b, T_{\beta}\right] f(x)-\left(\left[b, T_{\beta}\right] f\right)_{B}\right| d x \\
& \quad \lesssim \frac{1}{|B|} \int_{B}\left|B_{1}(x)-\left(B_{1}\right)_{B}\right| d x+\frac{1}{|B|} \int_{B}\left|B_{2}(x)-\left(B_{2}\right)_{B}\right| d x \\
& \quad=: A_{1}+A_{2},
\end{aligned}
$$

where

$$
A_{1}=\frac{1}{|B|} \int_{B}\left|B_{1}(x)-\left(B_{1}\right)_{B}\right| d x
$$


and

$$
A_{2}=\frac{1}{|B|} \int_{B}\left|B_{2}(x)-\left(B_{2}\right)_{B}\right| d x .
$$

For $A_{1}$, let $p>\frac{s}{s-\beta}$. Because $r<\gamma \rho\left(x_{0}\right)$, by Hölder's inequality and Lemma 2.9, we have

$$
A_{1} \lesssim\|b\|_{\sigma} M_{p}\left(T_{\beta} f\right)(x)
$$

For $A_{2}$, let $x \in \mathbb{R}^{n}$ and $B=B\left(x_{0}, r\right)$ with $r<\gamma \rho\left(x_{0}\right)$ such that $x \in B$. We split $f=f_{1}+f_{2}$ with $f_{1}=f \chi_{2 B}$. Hence we can divide $A_{2}$ into two parts as

$$
A_{2}=\frac{1}{|B|} \int_{B}\left|B_{2}(x)-\left(B_{2}\right)_{B}\right| d x=: A_{2,1}+A_{2,2},
$$

where

$$
A_{2,1}=\frac{1}{|B|} \int_{B}\left|T_{\beta}\left(\left(b-b_{B}\right) f_{1}\right)(x)-\left(T_{\beta}\left(b-b_{B}\right) f_{1}\right)_{B}\right| d x
$$

and

$$
A_{2,2}=\frac{1}{|B|} \int_{B}\left|T_{\beta}\left(\left(b-b_{B}\right) f_{2}\right)(x)-\left(T_{\beta}\left(b-b_{B}\right) f_{2}\right)_{B}\right| d x .
$$

Take $p_{0} \in\left(\frac{s}{s-\beta}, p\right)$ and let $\mu=\frac{p}{p-p_{0}}$. Applying Corollary 3.4 and Hölder's inequality, we can get

$$
\begin{aligned}
A_{2,1} & \lesssim \frac{1}{|B|} \int_{B}\left|T_{\beta}\left(\left(b-b_{B}\right) f_{1}\right)(x)\right| d x \\
& \lesssim\left\{\frac{1}{|B|} \int_{B}\left|T_{\beta}\left(\left(b-b_{B}\right) f_{1}\right)(x)\right|^{p_{0}} d x\right\}^{\frac{1}{p_{0}}} \\
& \lesssim\left\{\frac{1}{|B|} \int_{2 B}\left|\left(b(x)-b_{B}\right) f_{1}(x)\right|^{p_{0}} d x\right\}^{\frac{1}{p_{0}}} \\
& \lesssim\left\{\frac{1}{|B|} \int_{2 B}\left|b(x)-b_{B}\right|^{\mu p_{0}} d x\right\}^{\frac{1}{\mu p_{0}}}\left\{\frac{1}{|B|} \int_{2 B}|f(x)|^{p} d x\right\}^{\frac{1}{p}} \\
& \lesssim\|b\|_{\sigma} M_{p}(f)(x) .
\end{aligned}
$$

For $A_{2,2}$, by Lemma 3.7, we have

$$
\begin{aligned}
A_{2,2} & \lesssim \frac{1}{|B|^{2}} \iint_{B}\left|T_{\beta}\left(\left(b-b_{B}\right) f_{2}\right)(u)-T_{\beta}\left(\left(b-b_{B}\right) f_{2}\right)(y)\right| d u d y \\
& \lesssim\|b\|_{\sigma} M_{p}(f)(x) .
\end{aligned}
$$

Therefore, we get

$$
\left|M_{\rho, \gamma}^{\sharp}\left(\left[b, T_{\beta}\right] f\right)(x)\right| d x \lesssim\|b\|_{\sigma}\left\{M_{p}\left(T_{\beta} f\right)(x)+M_{p}(f)(x)\right\} .
$$


Finally, by the $L^{p}\left(\mathbb{R}^{n}\right)$ boundedness of $M_{p}$, we obtain

$$
\left\|M_{\rho, \gamma}^{\sharp}\left(\left[b, T_{\beta}\right] f\right)\right\|_{L^{q}} \lesssim\|b\|_{\sigma}\|f\|_{L^{q}} .
$$

This completes the proof of Theorem 3.8.

\subsection{The boundedness of $\left[b, T_{\beta}\right]$ on Herz spaces}

Theorem 3.9 Suppose that $V \in B_{s}, s \geq \frac{n}{2}$. Let $0<\beta<\frac{n}{2}, 0<p<\infty$, and $\frac{s}{s-\beta}<q<\infty$. If $-\frac{n}{q}<\alpha<n\left(1-\frac{1}{q}-\frac{2 \beta}{n}\right)$, then

$$
\left\|T_{\beta} f\right\|_{\dot{K}_{q}^{\alpha, p}\left(\mathbb{R}^{n)}\right.} \lesssim\|f\|_{\dot{K}_{q}^{\alpha, p}\left(\mathbb{R}^{n}\right)}
$$

Proof We write

$$
f(y)=\sum_{j=-\infty}^{\infty} f(y) \chi_{E_{j}}(y)=\sum_{j=-\infty}^{\infty} f_{j}(y),
$$

which gives

$$
\begin{aligned}
\left\|T_{\beta} f\right\|_{\dot{K}_{q}^{\alpha, p}\left(\mathbb{R}^{n)}\right)}^{p} & \sum_{k=-\infty}^{\infty} 2^{k \alpha p}\left(\sum_{j=-\infty}^{k-2}\left\|\chi_{k} T_{\beta} f_{j}\right\|_{L^{q}\left(\mathbb{R}^{n)}\right)}\right)^{p} \\
& +\sum_{k=-\infty}^{\infty} 2^{k \alpha p}\left(\sum_{j=k-1}^{k+1}\left\|\chi_{k} T_{\beta} f_{j}\right\|_{L^{q}\left(\mathbb{R}^{n}\right)}\right)^{p} \\
& +\sum_{k=-\infty}^{\infty} 2^{k \alpha p}\left(\sum_{j=k+2}^{\infty}\left\|\chi_{k} T_{\beta} f_{j}\right\|_{L^{q}\left(\mathbb{R}^{n}\right)}\right)^{p} \\
= & M_{1}+M_{2}+M_{3} .
\end{aligned}
$$

For $M_{2}$, by Corollary 3.4, we have

$$
M_{2} \lesssim \sum_{k=-\infty}^{\infty} 2^{k \alpha p}\left(\sum_{j=k-1}^{k+1}\left\|f_{j}\right\|_{L^{q}\left(\mathbb{R}^{n}\right)}\right)^{p} \lesssim\|f\|_{\dot{K}_{q}^{\alpha, p}\left(\mathbb{R}^{n}\right)}^{p}
$$

Now we estimate $M_{1}$. Via a simple computation, for $x \in E_{k}$ and $y \in E_{j}$ with $j \leq k-2$, we can deduce that $|x-y| \sim 2^{k}$. By Lemmas $2.17,2.16$, and 2.6, we use Hölder's inequality to obtain

$$
\begin{aligned}
\left\|\chi_{k} T_{\beta} f_{j}\right\|_{L^{q}\left(\mathbb{R}^{n}\right)} & =\left(\int_{E_{k}}\left|\int_{E_{j}} K(x, y) f(y) d y\right|^{q} d x\right)^{\frac{1}{q}} \\
& \lesssim \frac{1}{\left(1+2^{k} / \rho(0)\right)^{N /\left(l_{0}+1\right)}} \frac{1}{\left(2^{k}\right)^{n-2 \beta}}\left(\int_{E_{k}}\left|\int_{E_{j}}\right| V^{\beta}(y) f(y)|d y|^{q} d x\right)^{\frac{1}{q}} \\
& \lesssim \frac{\left(\int_{E_{j}} V(y) d y\right)^{\beta}}{\left(1+2^{k} / \rho(0)\right)^{N /\left(l_{0}+1\right)}} \frac{\left|E_{j}\right|^{1-\frac{1}{q}-\beta}}{\left|E_{k}\right|^{1-\frac{1}{q}-\frac{2 \beta}{n}}} \mid f_{j} \|_{L^{q}\left(\mathbb{R}^{n}\right)} \\
& \lesssim \frac{\mid f_{j} \|_{L^{q}\left(\mathbb{R}^{n}\right)}}{\left(1+2^{k} / \rho(0)\right)^{N /\left(l_{0}+1\right)-N_{0} \beta}} \frac{\left|E_{j}\right|^{1-\frac{1}{q}-\frac{2 \beta}{n}}}{\left|E_{k}\right|^{1-\frac{1}{q}-\frac{2 \beta}{n}}} .
\end{aligned}
$$


Take $N$ large enough. For $-\frac{n}{q}<\alpha<n\left(-\frac{1}{q}+1-\frac{2 \beta}{n}\right)$, we have

$$
\begin{aligned}
M_{1} & \lesssim \sum_{k=-\infty}^{\infty} 2^{k \alpha p}\left\{\sum_{j=-\infty}^{k-2} 2^{(j-k) n\left(1-\frac{1}{q}-\frac{2 \beta}{n}\right)}\left\|f_{j}\right\|_{L^{q\left(\mathbb{R}^{n}\right)}}\right\}^{p} \\
& \lesssim \sum_{k=-\infty}^{\infty}\left\{\sum_{j=-\infty}^{k-2} 2^{(j-k) n\left(1-\frac{1}{q}-\frac{2 \beta}{n}-\frac{\alpha}{n}\right)} 2^{j \alpha}\left\|f_{j}\right\|_{L^{q\left(\mathbb{R}^{n}\right)}}\right\}^{p}
\end{aligned}
$$

We divide the estimate of $M_{1}$ into two cases.

Case $1: p \leq 1$. By the $p$-triangle inequality

$$
\left(\sum_{k=-\infty}^{\infty}\left|a_{k}\right|\right)^{r} \leq \sum_{k=-\infty}^{\infty}\left|a_{k}\right|^{r}, \quad 0<r<1
$$

we have

$$
\begin{aligned}
M_{1} & \lesssim \sum_{k=-\infty}^{\infty} \sum_{j=-\infty}^{k-2} 2^{(j-k) n p\left(1-\frac{1}{q}-\frac{2 \beta}{n}-\frac{\alpha}{n}\right)} 2^{j \alpha p}\left\|f_{j}\right\|_{L^{q}\left(\mathbb{R}^{n}\right)}^{p} \\
& \lesssim \sum_{j=-\infty}^{\infty} 2^{j \alpha p}\left\|f_{j}\right\|_{L^{q}\left(\mathbb{R}^{n}\right)}^{p} \\
& \lesssim\|f\|_{\dot{K}_{q}^{\alpha, p}\left(\mathbb{R}^{n}\right)}^{p}
\end{aligned}
$$

Case 2: $p>1$. By the Hölder inequality, we can get

$$
\begin{aligned}
M_{1} \lesssim & \sum_{k=-\infty}^{\infty}\left\{\left(\sum_{j=-\infty}^{k-2} 2^{\frac{1}{2}(j-k) n p^{\prime}\left(1-\frac{1}{q}-\frac{2 \beta}{n}-\frac{\alpha}{n}\right)}\right)^{\frac{1}{p^{\prime}}}\right. \\
& \left.\times\left(\sum_{j=-\infty}^{k-2} 2^{\frac{1}{2}(j-k) n p\left(1-\frac{1}{q}-\frac{2 \beta}{n}-\frac{\alpha}{n}\right)} 2^{j \alpha p}\left\|f_{j}\right\|_{L^{q}\left(\mathbb{R}^{n}\right)}^{p}\right)^{\frac{1}{p}}\right\}^{p} \\
\lesssim & \sum_{j=-\infty}^{\infty} 2^{j \alpha p} \sum_{k=j+2}^{\infty} 2^{\frac{1}{2}(j-k) n p\left(1-\frac{1}{q}-\frac{2 \beta}{n}-\frac{\alpha}{n}\right)}\left\|f_{j}\right\|_{L^{q}\left(\mathbb{R}^{n}\right)}^{p} \\
\lesssim & \|f\|_{\dot{K}_{q}^{\alpha, p}}^{p}
\end{aligned}
$$

For $M_{3}$, note that when $x \in E_{k}, y \in E_{j}$, and $j \geq k+2$, then $|x-y| \sim 2^{j}$. Similar to $M_{1}$, we have

$$
\begin{aligned}
\left\|\chi_{k} T_{\beta} f_{j}\right\|_{L^{q}\left(\mathbb{R}^{n}\right)} & \lesssim \frac{1}{\left(1+2^{j} / \rho(0)\right)^{N /\left(l_{0}+1\right)}} \frac{1}{\left(2^{j}\right)^{n-2 \beta}}\left(\int_{E_{k}}\left|\int_{E_{j}}\right| V^{\beta} f(y)|d y|^{q} d x\right)^{\frac{1}{q}} \\
& \lesssim \frac{\left|E_{k}\right|^{\frac{1}{q}}\left|E_{j}\right|^{-\frac{1}{q}}\left\|f_{j}\right\|_{L^{q}\left(\mathbb{R}^{n}\right)}}{\left(1+2^{j} / \rho(0)\right)^{N /\left(l_{0}+1\right)-N_{0} \beta}} .
\end{aligned}
$$


Because $-\frac{n}{q}<\alpha<n\left(-\frac{1}{q}+1-\frac{2 \beta}{n}\right)$, we use (3.1) and Hölder's inequality to obtain

$$
M_{3} \lesssim \sum_{k=-\infty}^{\infty}\left\{\sum_{j=k+2}^{\infty} 2^{(k-j) n\left(\frac{1}{q}+\frac{\alpha}{n}\right)} 2^{j \alpha}\left\|f_{j}\right\|_{L^{q}\left(\mathbb{R}^{n}\right)}\right\}^{p} \lesssim\|f\|_{\dot{K}_{q}^{\alpha, p}\left(\mathbb{R}^{n}\right)}^{p}
$$

Finally, we get

$$
\left\|T_{\beta} f\right\|_{\dot{K}_{q}^{\alpha, p}\left(\mathbb{R}^{n}\right)} \lesssim\|f\|_{\dot{K}_{q}^{\alpha, p}\left(\mathbb{R}^{n}\right)}
$$

Theorem 3.10 Suppose that $V \in B_{s}, s>\frac{n}{2}$, and $b \in \operatorname{BMO}_{\sigma}(\rho), 0<\sigma<\infty$. If $0<\beta<\frac{n}{2}$, $0<p<\infty$, and $\frac{s}{s-\beta}<q<\infty$ with $-\frac{n}{q}<\alpha<n\left(1-\frac{1}{q}-\frac{2 \beta}{n}\right)$, then

$$
\left\|\left[b, T_{\beta}\right] f\right\|_{\dot{K}_{q}^{\alpha, p}\left(\mathbb{R}^{n}\right)} \lesssim\|b\|_{\sigma}\|f\|_{\dot{K}_{q}^{\alpha, p}\left(\mathbb{R}^{n}\right)} .
$$

Proof We write

$$
f(y)=\sum_{j=-\infty}^{\infty} f(y) \chi_{E_{j}}(y)=\sum_{j=-\infty}^{\infty} f_{j}(y)
$$

Hence, we have

$$
\begin{aligned}
\left\|\left[b, T_{\beta}\right] f\right\|_{\dot{K}_{q}^{\alpha, p}\left(\mathbb{R}^{n}\right)}^{p \lesssim} & \sum_{k=-\infty}^{\infty} 2^{k \alpha p}\left(\sum_{j=-\infty}^{k-2}\left\|\chi_{k}\left[b, T_{\beta}\right] f_{j}\right\|_{L^{q}\left(\mathbb{R}^{n}\right)}\right)^{p} \\
& +\sum_{k=-\infty}^{\infty} 2^{k \alpha p}\left(\sum_{j=k-1}^{k+1}\left\|\chi_{k}\left[b, T_{\beta}\right] f_{j}\right\|_{L^{q}\left(\mathbb{R}^{n}\right)}\right)^{p} \\
& +\sum_{k=-\infty}^{\infty} 2^{k \alpha p}\left(\sum_{j=k+2}^{\infty}\left\|\chi_{k}\left[b, T_{\beta}\right] f_{j}\right\|_{L^{q}\left(\mathbb{R}^{n}\right)}\right)^{p} \\
= & \widetilde{M}_{1}+\widetilde{M}_{2}+\tilde{M}_{3} .
\end{aligned}
$$

For $\widetilde{M}_{2}$, by Theorem 3.8 , we have

$$
\tilde{M}_{2} \lesssim \sum_{k=-\infty}^{\infty} 2^{k \alpha p}\left(\sum_{j=k-1}^{k+1}\left\|f_{j}\right\|_{L^{q}\left(\mathbb{R}^{n}\right)}\right)^{p} \lesssim\|f\|_{\dot{K}_{q}^{\alpha, p}\left(\mathbb{R}^{n}\right)}^{p}
$$

Now we estimate $\tilde{M}_{1}$. We write $b(x)-b(y)=\left(b(x)-b_{B_{j}}\right)-\left(b(y)-b_{B_{j}}\right)$ and get

$$
\begin{aligned}
\left\|\chi_{k}\left[b, T_{\beta}\right] f_{j}\right\|_{L^{q}\left(\mathbb{R}^{n}\right)}= & \left(\int_{E_{k}}\left|\int_{E_{j}}(b(x)-b(y)) K_{\beta}(x, y) f(y) d y\right|^{q} d x\right)^{\frac{1}{q}} \\
\lesssim & \left(\int_{E_{k}}\left|\left(b(x)-b_{B_{j}}\right) T_{\beta}\left(f_{j}\right)(x)\right|^{q} d x\right)^{\frac{1}{q}} \\
& +\left|E_{k}\right|^{\frac{1}{q}}\left\|T_{\beta}\left(\left(b-b_{B_{j}}\right) f_{j}\right)\right\|_{L^{q}\left(E_{k}\right)} \\
\lesssim & \widetilde{M}_{1,1}+\widetilde{M}_{1,2} .
\end{aligned}
$$


Because $x \in E_{k}, y \in E_{j}$, and $j \leq k-2$, we deduce that $|x-y| \sim 2^{k}$. By Lemmas 2.16 and 2.17, we have

$$
T_{\beta}\left(f_{j}\right)(x) \lesssim \frac{1}{\left(1+2^{k} / \rho(0)\right)^{N /\left(l_{0}+1\right)}} \frac{1}{\left(2^{k}\right)^{n-2 \beta}} \int_{E_{j}} V^{\beta}(y)|f(y)| d y .
$$

Using Lemmas 2.6 and 2.10 and the Hölder inequality, we have

$$
\begin{aligned}
\tilde{M}_{1,1} & \lesssim \frac{\left(\int_{E_{k}}\left|b(x)-b_{B_{j}}\right|^{q} d x\right)^{\frac{1}{q}}}{\left(1+2^{k} / \rho(0)\right)^{N /\left(l_{0}+1\right)}} \frac{1}{\left(2^{k}\right)^{n-2 \beta}} \int_{E_{j}} V^{\beta}(y)|f(y)| d y \\
& \lesssim \frac{(k-j)\|b\|_{\sigma}\left(\int_{E_{j}} V(y) d y\right)^{\beta}}{\left(1+2^{k} / \rho(0)\right)^{N /\left(l_{0}+1\right)-\left(l_{0}+1\right) \sigma}} \frac{\left|E_{j}\right|^{1-\frac{1}{q}-\beta}}{\left|E_{k}\right|^{1-\frac{1}{q}-\frac{2 \beta}{n}}}\left\|f_{j}\right\|_{L^{q}\left(\mathbb{R}^{n}\right)} \\
& \lesssim \frac{(k-j) 2^{(j-k) n\left(1-\frac{1}{q}-\frac{2 \beta}{n}\right)}\|b\|_{\sigma}\left\|f_{j}\right\|_{L^{q}\left(\mathbb{R}^{n}\right)}}{\left(1+2^{k} / \rho(0)\right)^{N /\left(l_{0}+1\right)-\left(l_{0}+1\right) \sigma-N_{0} \beta}} .
\end{aligned}
$$

For the term $\widetilde{M}_{1,2}$, we use Hölder's inequality to obtain

$$
\begin{aligned}
& \int_{E_{j}}\left|b(y)-b_{B_{j}}\right| V^{\beta}(y)|f(y)| d y \\
& \quad \lesssim\|b\|_{\sigma}\left(1+2^{j} / \rho(0)\right)^{\left(l_{0}+1\right) \sigma}\left\|f_{j}\right\|_{L^{q}\left(\mathbb{R}^{n)}\right.}\left(\frac{1}{\left|E_{j}\right|} \int_{E_{j}}|V(y)|^{s} d y\right)^{\frac{\beta}{s}}\left|E_{j}\right|^{1-\frac{1}{q}} \\
& \quad \lesssim\|b\|_{\sigma}\left(1+2^{j} / \rho(0)\right)^{\left(l_{0}+1\right) \sigma}\left\|f_{j}\right\|_{L^{q}\left(\mathbb{R}^{n}\right)}\left(\int_{E_{j}}|V(y)| d y\right)^{\beta}\left|E_{j}\right|^{1-\frac{1}{q}-\beta}
\end{aligned}
$$

which gives

$$
\left|T_{\beta}\left(\left(b-b_{B_{j}}\right) f\right)(x)\right| \lesssim \frac{\|b\|_{\sigma}\left\|f_{j}\right\|_{L^{q}\left(\mathbb{R}^{n}\right)}\left|E_{j}\right|^{1-\frac{1}{q}-\frac{2 \beta}{n}}}{\left(1+2^{j} / \rho(0)\right)^{-\left(l_{0}+1\right) \sigma-N_{0} \beta}} .
$$

The above estimate implies that

$$
\begin{aligned}
\widetilde{M}_{1,2} & \lesssim \frac{1}{\left(1+2^{k} / \rho(0)\right)^{N /\left(l_{0}+1\right)}} \frac{\left|E_{k}\right|^{\frac{1}{q}}}{\left(2^{k}\right)^{n-2 \beta}} \frac{\|b\|_{\sigma}\left\|f_{j}\right\|_{L^{q}\left(\mathbb{R}^{n}\right)}\left|E_{j}\right|^{1-\frac{1}{q}-\frac{2 \beta}{n}}}{\left(1+2^{j / \rho} /(0)\right)^{-\left(l_{0}+1\right) \sigma-N_{0} \beta}} \\
& \lesssim \frac{2^{(j-k) n\left(1-\frac{1}{q}-\frac{2 \beta}{n}\right)}\|b\|_{\sigma}\left\|f_{j}\right\|_{L^{q}\left(\mathbb{R}^{n}\right)}}{\left(1+2^{k} / \rho(0)\right)^{N /\left(l_{0}+1\right)-\left(l_{0}+1\right) \sigma-N_{0} \beta}} .
\end{aligned}
$$

Take $N$ large enough. Because $-\frac{n}{q}<\alpha<n\left(-\frac{1}{q}+1-\frac{2 \beta}{n}\right)$, we obtain

$$
\begin{aligned}
\widetilde{M}_{1} & \lesssim\|b\|_{\sigma}^{p} \sum_{k=-\infty}^{\infty}\left\{\sum_{j=-\infty}^{k-2}(k-j) 2^{(j-k) n\left(1-\frac{1}{q}-\frac{2 \beta}{n}\right)}\left\|f_{j}\right\|_{L^{q}\left(\mathbb{R}^{n}\right)}\right\}^{p} \\
& \lesssim\|b\|_{\sigma}^{p} \sum_{k=-\infty}^{\infty}\left\{\sum_{j=-\infty}^{k-2}(k-j) 2^{(j-k) n\left(1-\frac{1}{q}-\frac{2 \beta}{n}-\frac{\alpha}{n}\right)} 2^{j \alpha}\left\|f_{j}\right\|_{L q}\left(\mathbb{R}^{n)}\right\}^{p} .\right.
\end{aligned}
$$

We still divide the proof into two cases. 
Case 1: $p \leq 1$. By the inequality (3.1) we have

$$
\begin{aligned}
\widetilde{M}_{1} & \lesssim\|b\|_{\sigma}^{p} \sum_{k=-\infty}^{\infty} \sum_{j=-\infty}^{k-2}(k-j)^{p} 2^{(j-k) n p\left(1-\frac{1}{q}-\frac{2 \beta}{n}-\frac{\alpha}{n}\right)} 2^{j \alpha p}\left\|f_{j}\right\|_{L^{q}\left(\mathbb{R}^{n}\right)}^{p} \\
& \lesssim\|b\|_{\sigma}^{p} \sum_{j=-\infty}^{\infty} 2^{j \alpha p}\left\|f_{j}\right\|_{L^{q}\left(\mathbb{R}^{n}\right)}^{p} \\
& \lesssim\|b\|_{\sigma}^{p}\|f\|_{\dot{K}_{q}^{\alpha, p}\left(\mathbb{R}^{n}\right)}^{p} .
\end{aligned}
$$

Case 2: $p>1$. The Hölder inequality implies that

$$
\begin{aligned}
& \tilde{M}_{1} \lesssim\|b\|_{\sigma}^{p} \sum_{k=-\infty}^{\infty}\left\{\left(\sum_{j=-\infty}^{k-2}(k-j)^{p^{\prime}} 2^{\frac{1}{2}(j-k) n p^{\prime}\left(1-\frac{1}{q}-\frac{2 \beta}{n}-\frac{\alpha}{n}\right)}\right)^{\frac{1}{p^{\prime}}}\right. \\
&\left.\times\left(\sum_{j=-\infty}^{k-2} 2^{\frac{1}{2}(j-k) n p\left(1-\frac{1}{q}-\frac{2 \beta}{n}-\frac{\alpha}{n}\right)} 2^{j \alpha p}\left\|f_{j}\right\|_{L^{q}\left(\mathbb{R}^{n}\right)}^{p}\right)^{\frac{1}{p}}\right\}^{p} \\
& \lesssim\|b\|_{\sigma}^{p} \sum_{j=-\infty}^{\infty} 2^{j \alpha p} \sum_{k=j+2}^{\infty} 2^{\frac{1}{2}(j-k) n p\left(1-\frac{1}{q}-\frac{2 \beta}{n}-\frac{\alpha}{n}\right)}\left\|f_{j}\right\|_{L^{q}\left(\mathbb{R}^{n}\right)}^{p} \\
& \lesssim\|b\|_{\sigma}^{p}\|f\|_{\dot{K}_{q}^{\alpha, p}\left(\mathbb{R}^{n}\right)}^{p}
\end{aligned}
$$

For $\widetilde{M}_{3}$, note that when $x \in E_{k}, y \in E_{j}$, and $j \geq k+2$, then $|x-y| \sim 2^{j}$. Similar to Theorem 3.9, using the following decomposition: $b(x)-b(y)=\left(b(x)-b_{B_{k}}\right)-\left(b(y)-b_{B_{k}}\right)$, we can get

$$
\begin{aligned}
& \left\|\chi_{k}\left[b, T_{\beta}\right] f_{j}\right\|_{L^{q}\left(\mathbb{R}^{n}\right)} \\
& \quad \lesssim \frac{1}{\left(1+2^{j} / \rho(0)\right)^{N /\left(l_{0}+1\right)}} \frac{1}{\left(2^{j}\right)^{n-2 \beta}}\left(\int_{E_{k}}\left[\int_{E_{j}}|b(x)-b(y)| V^{\beta}|f(y)| d y\right]^{q} d x\right)^{\frac{1}{q}} \\
& \quad \lesssim \frac{(j-k)\|b\|_{\sigma}}{\left(1+2^{j} / \rho(0)\right)^{N /\left(l_{0}+1\right)-\left(l_{0}+1\right) \sigma-N_{0} \beta}}\left|E_{k}\right|^{\frac{1}{q}}\left|E_{j}\right|^{-\frac{1}{q}}\left\|f_{j}\right\|_{L^{q}\left(\mathbb{R}^{n}\right)} .
\end{aligned}
$$

Since $N$ is large enough and $-\frac{n}{q}<\alpha<n\left(-\frac{1}{q}+1-\frac{2 \beta}{n}\right)$, we obtain

$$
\widetilde{M}_{3} \lesssim\|b\|_{\sigma}^{p} \sum_{k=-\infty}^{\infty}\left\{\sum_{j=k+2}^{\infty}(j-k) 2^{(k-j) n\left(\frac{1}{q}+\frac{\alpha}{n}\right)} 2^{j \alpha}\left\|f_{j}\right\|_{L^{q}\left(\mathbb{R}^{n}\right)}\right\}^{p} .
$$

Similar to $\widetilde{M}_{1}$, we have $\widetilde{M}_{3} \lesssim\|b\|_{\sigma}^{p}\|f\|_{\dot{K}_{q}^{\alpha, p}\left(\mathbb{R}^{n}\right)}^{p}$. Finally, we get

$$
\left\|\left[b, T_{\beta}\right] f\right\|_{\dot{K}_{q}^{\alpha, p}\left(\mathbb{R}^{n}\right)} \lesssim\|b\|_{\sigma}\|f\|_{\dot{K}_{q}^{\alpha, p}\left(\mathbb{R}^{n}\right)} .
$$

Let $T_{\beta}^{\prime}$ be the dual operator of $T_{\beta}$. By the duality of Herz spaces, we have the following corollary of Theorem 3.10 .

Corollary 3.11 Suppose that $V \in B_{s}, s \geq \frac{n}{2}$, and $b \in \mathrm{BMO}_{\sigma}(\rho), 0<\sigma<\infty$. If $0<\beta<\frac{n}{2}$, $0<p<\infty, 1<q<\frac{s}{\beta}$ with $n\left(-\frac{1}{q}+\frac{2 \beta}{n}\right)<\alpha<n\left(1-\frac{1}{q}\right)$, then 
(a) $\left\|T_{\beta}^{\prime} f\right\|_{\dot{K}_{q}^{\alpha, p}\left(\mathbb{R}^{n}\right)} \lesssim\|f\|_{\dot{K}_{q}^{\alpha, p}\left(\mathbb{R}^{n}\right)}$;

(b) $\left\|\left[b, T_{\beta}^{\prime}\right] f\right\|_{\dot{K}_{q}^{\alpha, p}\left(\mathbb{R}^{n}\right)} \lesssim\|b\|_{\sigma}\|f\|_{\dot{K}_{q}^{\alpha, p}\left(\mathbb{R}^{n}\right)}$.

With the help of Lemma 2.12, we can get some similar results to Theorem 3.9, 3.10 and Corollary 3.11 for the non-homogeneous Herz spaces $K_{q}^{\alpha, p}\left(\mathbb{R}^{n}\right)$.

Theorem 3.12 Suppose that $V \in B_{s}, s \geq \frac{n}{2}$ and $b \in \mathrm{BMO}_{\sigma}(\rho), 0<\sigma<\infty$. Let $0<\beta<\frac{n}{2}$ and $0<p<\infty$.

(1) If $\frac{s}{s-\beta}<q<\infty,-\frac{n}{q}<\alpha<n\left(1-\frac{1}{q}-\frac{2 \beta}{n}\right)$, then

$$
\left\{\begin{array}{l}
\left\|T_{\beta} f\right\|_{K_{q}^{\alpha, p}\left(\mathbb{R}^{n)}\right.} \lesssim\|f\|_{K_{q}^{\alpha, p}\left(\mathbb{R}^{n}\right)}, \\
\left\|\left[b, T_{\beta}\right] f\right\|_{K_{q}^{\alpha, p}\left(\mathbb{R}^{n}\right)} \lesssim\|b\|_{\sigma}\|f\|_{K_{q}^{\alpha, p}\left(\mathbb{R}^{n}\right)} .
\end{array}\right.
$$

(2) If $1<q<\frac{s}{\beta}, n\left(-\frac{1}{q}+\frac{2 \beta}{n}\right)<\alpha<n\left(1-\frac{1}{q}\right)$, then

$$
\left\{\begin{array}{l}
\left\|T_{\beta}^{\prime} f\right\|_{K_{q}^{\alpha, p}\left(\mathbb{R}^{n}\right)} \lesssim\|f\|_{K_{q}^{\alpha, p}\left(\mathbb{R}^{n)}\right)}, \\
\left\|\left[b, T_{\beta}^{\prime}\right] f\right\|_{K_{q}^{\alpha, p}\left(\mathbb{R}^{n}\right)} \lesssim\|b\|_{\sigma}\|f\|_{K_{q}^{\alpha, p}\left(\mathbb{R}^{n}\right)} .
\end{array}\right.
$$

\section{Commutators $\left[b, R_{L}\right]$ on Herz type spaces}

In this section, let $R_{L}=\nabla(-\Delta+V)^{-1 / 2}$ be the Riesz transform associated to Schrödinger operators $L=-\Delta+V$ with $V \in B_{s}, s>n / 2$. For $b \in \operatorname{BMO}_{\sigma}(\rho)$, we consider the boundedness of $R_{L}$ and the commutator $\left[b, R_{L}\right]$ on the Herz spaces, respectively. Denote by $K_{R_{L}}$ the kernel of $R_{L}$, that is,

$$
R_{L}(f)(x)=\int K_{R_{L}}(x, y) f(y) d y .
$$

By the fundamental solution of $L$, Shen [1] obtained the following estimate of $K_{R_{L}}$.

Lemma 4.1 ([1], Section 5) Suppose $V \in B_{s}, s>\frac{n}{2}$. Then for every $N$, there exists a constant $C_{N}>0$ such that

$$
\left|K_{R_{L}}(x, y)\right| \leq \frac{C_{N}}{(1+|x-y| / \rho(x))^{N}}\left(\frac{1}{|x-y|^{n-1}} \int_{B(y,|x-y|)} \frac{V(z)}{|z-y|^{n-1}} d z d y+\frac{1}{|x-y|^{n}}\right) .
$$

Let $R_{L}^{\prime}=(-\Delta+V)^{-1 / 2} V^{1 / 2}$ be the dual operator of $R_{L}$. By Lemma 4.1, Shen proved that the operator $R_{L}$ is bounded on $L^{p}\left(\mathbb{R}^{n}\right)$. Precisely, we have the following.

Lemma 4.2 ([1], Theorem 0.5) Suppose that $V \in B_{s}$ for some $s>\frac{n}{2}$, and let $\left(\frac{1}{s}-\frac{1}{n}\right)^{+}=\frac{1}{\tilde{q}}$. Then

(1) $R_{L}$ is bounded on $L^{q}\left(\mathbb{R}^{n}\right)$ for $1<q<\tilde{q}$;

(2) $R_{L}^{\prime}$ is bounded on $L^{q}\left(\mathbb{R}^{n}\right)$ for $\tilde{q}<q<\infty$.

By the idea of Shen [1], Guo, Li, and Peng [9] obtained a regularity estimate of the kernel $R_{L}$ and proved the commutators $\left[b, R_{L}\right]$ are bounded on some Lebesgue spaces $L^{p}$ with $b \in \mathrm{BMO}\left(\mathbb{R}^{n}\right)$. For $b \in \mathrm{BMO}_{\infty}(\rho)$, Bongioanni, Harboure, and Salinas [14] proved the $L^{p}$ boundedness of $\left[b, R_{L}\right]$. Recall that $\mathrm{BMO}\left(\mathbb{R}^{n}\right) \subset \mathrm{BMO}_{\sigma}(\rho)$. Hence the result of Bongioanni, Harboure, and Salinas [14] generalizes that of Guo, Li, and Peng [9]. 
Lemma 4.3 ([14], Theorem 1) Suppose that $V \in B_{s}$ for some $s>\frac{n}{2}$, and $b \in \mathrm{BMO}_{\infty}(\rho)$. Let $\left(\frac{1}{s}-\frac{1}{n}\right)^{+}=\frac{1}{\tilde{q}}$, then

(1) $\left[b, R_{L}\right]$ is bounded on $L^{q}\left(\mathbb{R}^{n}\right)$ for $1<q<\tilde{q}$;

(2) $\left[b, R_{L}^{\prime}\right]$ is bounded on $L^{q}\left(\mathbb{R}^{n}\right)$ for $\tilde{q}<q<\infty$.

Based on the results of Shen [1] and Bongioanni, Harboure, and Salinas [14], we consider the boundedness of $R_{L}$ on Herz spaces. If $s \geq n$, Shen [1] proved that $R_{L}$ is a CalderónZygmund operator. By Theorem 6.2.1 in [20], it is obvious that $R_{L}$ is bounded on Herz spaces. Hence in the next theorem, we only consider the case of $\frac{n}{2}<s<n$.

Theorem 4.4 Suppose that $V \in B_{s}, s>\frac{n}{2}$, and let $\left(\frac{1}{s}-\frac{1}{n}\right)^{+}=\frac{1}{\tilde{q}}$. If $0<p<\infty,(\tilde{q})^{\prime}<q<\infty$, and $-\frac{n}{q}<\alpha<n\left(1-\frac{1}{q}-\frac{1}{\tilde{q}}\right)$, then

$$
\left\|R_{L} f\right\|_{\dot{K}_{q}^{\alpha, p}\left(\mathbb{R}^{n)}\right.} \leq C\|f\|_{\dot{K}_{q}^{\alpha, p}\left(\mathbb{R}^{n}\right)}
$$

Proof By the decomposition

$$
f(y)=\sum_{j=-\infty}^{\infty} f(y) \chi_{E_{j}}(y)=\sum_{j=-\infty}^{\infty} f_{j}(y)
$$

we obtain

$$
\begin{aligned}
\left\|R_{L} f\right\|_{\dot{K}_{q}^{\alpha, p}\left(\mathbb{R}^{n}\right)}^{p} \lesssim & \sum_{k=-\infty}^{\infty} 2^{k \alpha p}\left(\sum_{j=-\infty}^{k-2}\left\|\chi_{k} R_{L} f_{j}\right\|_{L^{q}\left(\mathbb{R}^{n}\right)}\right)^{p} \\
& +\sum_{k=-\infty}^{\infty} 2^{k \alpha p}\left(\sum_{j=k-1}^{k+1}\left\|\chi_{k} R_{L} f_{j}\right\|_{L^{q}\left(\mathbb{R}^{n}\right)}\right)^{p} \\
& +\sum_{k=-\infty}^{\infty} 2^{k \alpha p}\left(\sum_{j=k+2}^{\infty}\left\|\chi_{k} R_{L} f_{j}\right\|_{L^{q}\left(\mathbb{R}^{n}\right)}\right)^{p} \\
= & W_{1}+W_{2}+W_{3} .
\end{aligned}
$$

For $W_{2}$, by Lemma 4.3 , we have

$$
W_{2} \lesssim \sum_{k=-\infty}^{\infty} 2^{k \alpha p}\left(\sum_{j=k-1}^{k+1}\left\|f_{j}\right\|_{L^{q}\left(\mathbb{R}^{n}\right)}\right)^{p} \lesssim\|f\|_{\dot{K}_{q}^{\alpha, p}\left(\mathbb{R}^{n}\right)}^{p}
$$

Now we estimate $W_{1}$. Because $x \in E_{k}, y \in E_{j}$, and $j \leq k-2$, we deduce that $|x-y| \sim 2^{k}$. By Lemmas 4.1 and 2.16, we get

$$
\begin{aligned}
& \left\|\chi_{k} R_{L} f_{j}\right\|_{L^{q}\left(\mathbb{R}^{n}\right)} \\
& \quad=\left(\int_{E_{k}}\left|\int_{E_{j}} K_{R_{L}}(x, y) f(y) d y\right|^{q} d x\right)^{\frac{1}{q}} \\
& \quad \lesssim\left(\int_{E_{k}}\left|\int_{E_{j}} \frac{|f(y)|}{(1+|x-y| / \rho(x))^{N}} \frac{1}{|x-y|^{n-1}} \int_{B(y,|x-y|)} \frac{V(z)}{|z-y|^{n-1}} d z d y\right|^{q} d x\right)^{\frac{1}{q}}
\end{aligned}
$$




$$
\begin{aligned}
& +\left(\int_{E_{k}}\left|\int_{E_{j}} \frac{|f(y)|}{(1+|x-y| / \rho(x))^{N}} \frac{1}{|x-y|^{n}} d y\right|^{q} d x\right)^{\frac{1}{q}} \\
= & W_{1,1}+W_{1,2} .
\end{aligned}
$$

For $W_{1,1}$, by Lemma 2.17 , we have

$$
\begin{aligned}
W_{1,1} & \lesssim \frac{1}{\left(1+2^{k} / \rho(0)\right)^{N /\left(l_{0}+1\right)}} \frac{1}{\left|2^{k}\right|^{n-1}}\left(\int_{E_{k}}\left|\int_{E_{j}}\right| f(y)\left|\int_{B(y,|x-y|)} \frac{V(z)}{|z-y|^{n-1}} d z d y\right|^{q} d x\right)^{\frac{1}{q}} \\
& \lesssim \frac{1}{\left(1+2^{k} / \rho(0)\right)^{N /\left(l_{0}+1\right)}} \frac{\left|E_{k}\right|^{\frac{1}{q}}}{\left|2^{k}\right|^{n-1}} \int_{E_{j}}|f(y)| \int_{B\left(0,2^{k+1}\right)} \frac{V(z)}{|z-y|^{n-1}} d z d y .
\end{aligned}
$$

Using the Hölder inequality, we obtain

$$
\begin{aligned}
& \int_{E_{j}}|f(y)| \int_{B\left(0,2^{k+1}\right)} \frac{V(z)}{|z-y|^{n-1}} d z d y \\
& \quad \lesssim\left|E_{j}\right|^{1-\frac{1}{q}-\frac{1}{\tilde{q}}}\left\|f_{j}\right\|_{L^{q}\left(\mathbb{R}^{n}\right)} \| \mathcal{I}_{1}\left(V \chi_{B\left(0,2^{k+1}\right)} \|_{L^{\tilde{q}\left(\mathbb{R}^{n}\right)}}\right. \\
& \quad \lesssim\left|E_{j}\right|^{1-\frac{1}{q}-\frac{1}{\tilde{q}}}\left\|f_{j}\right\|_{L^{q}\left(\mathbb{R}^{n}\right)}\left\|V \chi_{B\left(0,2^{k+1}\right)}\right\|_{L^{s}\left(\mathbb{R}^{n}\right)},
\end{aligned}
$$

where in the last inequality, we have used the boundedness of the fractional integral $\mathcal{I}_{1}$ with $\frac{1}{\tilde{q}}=\frac{1}{s}-\frac{1}{n}$. Because $V \in B_{s}$, we use Lemma 2.6 to deduce that

$$
\begin{aligned}
\left\|V \chi_{B\left(0,2^{k+1}\right)}\right\|_{L^{s}\left(\mathbb{R}^{n}\right)} & \lesssim\left|E_{k+1}\right|^{\frac{1}{s}-1} \int_{B\left(0,2^{k+1}\right)} V(z) d z \\
& \lesssim\left|E_{k+1}\right|^{\frac{1}{s}-1}\left(2^{k+1}\right)^{n-2}\left(1+2^{k+1} / \rho(0)\right)^{N_{0}} \\
& \lesssim\left|E_{k+1}\right|^{\frac{1}{s}-\frac{2}{n}}\left(1+2^{k+1} / \rho(0)\right)^{N_{0}} .
\end{aligned}
$$

The above estimate (4.2) implies that

$$
W_{1,1} \lesssim \frac{\left\|f_{j}\right\|_{L^{q}\left(\mathbb{R}^{n}\right)}}{\left(1+2^{k} / \rho(0)\right)^{N /\left(l_{0}+1\right)-N_{0}}} \frac{\left|E_{j}\right|^{1-\frac{1}{q}-\frac{1}{\bar{q}}}}{\left|E_{k}\right|^{1-\frac{1}{q}-\frac{1}{\bar{q}}}} .
$$

Let $N$ be large enough. When $j \leq k-2$, we have

$$
W_{1,1} \lesssim 2^{(j-k) n\left(1-\frac{1}{q}-\frac{1}{\tilde{q}}\right)}\left\|f_{j}\right\|_{L^{q}\left(\mathbb{R}^{n}\right)}
$$

Next we estimate $W_{1,2}$. We have

$$
\begin{aligned}
W_{1,2} & \lesssim \frac{1}{\left(1+2^{k} / \rho(0)\right)^{N /\left(l_{0}+1\right)}} \frac{1}{\left|2^{k}\right|^{n}}\left(\int_{E_{k}}\left|\int_{E_{j}}\right| f(y)|d y|^{q}\right)^{\frac{1}{q}} \\
& \lesssim \frac{\left\|f_{j}\right\|_{L^{q}\left(\mathbb{R}^{n}\right)\left|E_{j}\right|^{1-\frac{1}{q}}}}{\left(1+2^{k} / \rho(0)\right)^{N /\left(l_{0}+1\right)}} \frac{\left|E_{k}\right|^{\frac{1}{q}}}{\left|2^{k}\right|^{n}} .
\end{aligned}
$$

Take $N$ large enough. For $j \leq k-2$, we have

$$
W_{1,2} \lesssim 2^{(j-k) n\left(1-\frac{1}{q}\right)}\left\|f_{j}\right\|_{L^{q}\left(\mathbb{R}^{n}\right)} \lesssim 2^{(j-k) n\left(1-\frac{1}{q}-\frac{1}{q}\right)}\left\|f_{j}\right\|_{L^{q}\left(\mathbb{R}^{n}\right)} .
$$


Hence, when $j \leq k-2$, we have

$$
\left\|\chi_{k} R_{L} f_{j}\right\|_{L^{q}\left(\mathbb{R}^{n}\right)} \lesssim 2^{(j-k) n\left(1-\frac{1}{q}-\frac{1}{\tilde{q}}\right)}\left\|f_{j}\right\|_{L^{q}\left(\mathbb{R}^{n}\right)}
$$

Since $-\frac{n}{q}<\alpha<n\left(1-\frac{1}{q}-\frac{1}{\tilde{q}}\right)$, we have

$$
W_{1} \lesssim \sum_{k=-\infty}^{\infty} 2^{k \alpha p}\left(\sum_{j=-\infty}^{k-2} 2^{(j-k) n\left(1-\frac{1}{q}-\frac{1}{\tilde{q}}\right)}\left\|f_{j}\right\|_{L^{q}\left(\mathbb{R}^{n}\right)}\right)^{p}
$$

Similar to the proof of Theorem 3.10, by Hölder's inequality and (4.1), we obtain

$$
W_{1} \lesssim\|f\|_{\dot{K}_{q}^{\alpha, p}}^{p}
$$

For $W_{3}$, note that when $x \in E_{k}, y \in E_{j}$, and $j \geq k+2$, then $|x-y| \sim 2^{j}$. We have

$$
\int_{B(y,|x-y|)} \frac{V(z)}{|z-y|^{n-1}} d z \lesssim \int_{B\left(0, j^{j+1}\right)} \frac{V(z)}{|z-y|^{n-1}} d z
$$

Therefore, when $j \geq k+2$, by Lemma 2.17 , we have

$\left\|\chi_{k} R_{L} f_{j}\right\|_{L^{q}\left(\mathbb{R}^{n}\right)}$

$$
\begin{aligned}
\lesssim & \frac{1}{\left(1+2^{j} / \rho(0)\right)^{N /\left(l_{0}+1\right)}} \frac{1}{\left|2^{j}\right|^{n-1}}\left(\int_{E_{k}}\left|\int_{E_{j}}\right| f(y)\left|\int_{B\left(0,2^{j+1}\right)} \frac{V(z)}{|z-y|^{n-1}} d z d y\right|^{q} d x\right)^{\frac{1}{q}} \\
& +\frac{1}{\left(1+2^{j} / \rho(0)\right)^{N /\left(l_{0}+1\right)}} \frac{1}{\left|2^{j}\right|^{n}}\left(\int_{E_{k}}\left|\int_{E_{j}}\right| f(y)|d y|^{q} d x\right)^{\frac{1}{q}} \\
= & W_{3,1}+W_{3,2} .
\end{aligned}
$$

Similar to (4.1) and (4.2), we get

$$
\int_{E_{j}}|f(y)| \int_{B\left(0,2^{j+1}\right)} \frac{V(z)}{|z-y|^{n-1}} d z d y \lesssim\left|E_{j}\right|^{1-\frac{1}{q}-\frac{1}{\tilde{q}}}\left\|f_{j}\right\|_{L^{q}\left(\mathbb{R}^{n}\right)}\left\|V \chi_{B\left(0,2^{j+1}\right)}\right\|_{L^{s}\left(\mathbb{R}^{n}\right)}
$$

and

$$
\left\|V \chi_{B\left(0,2^{j+1}\right)}\right\|_{L^{s}\left(\mathbb{R}^{n}\right)} \lesssim\left|E_{j+1}\right|^{\frac{1}{s}-\frac{2}{n}}\left(1+2^{j+1} / \rho(0)\right)^{N_{0}}
$$

Take $N$ large enough. Because $\frac{1}{\tilde{q}}=\frac{1}{s}-\frac{1}{n}$, we have

$$
\begin{aligned}
W_{3,1} & \lesssim \frac{\left\|f_{j}\right\|_{L^{q}\left(\mathbb{R}^{n}\right)}}{\left(1+2^{k} / \rho(0)\right)^{N /\left(l_{0}+1\right)-N_{0}}} \frac{\left|E_{k}\right|^{\frac{1}{q}}}{\left|E_{j}\right|^{\left(1-\frac{1}{n}\right)-\left(1-\frac{1}{q}-\frac{1}{\tilde{q}}\right)-\left(\frac{1}{s}-\frac{2}{n}\right)}} \\
& \lesssim \frac{\left\|f_{j}\right\|_{L^{q}\left(\mathbb{R}^{n}\right)}}{\left(1+2^{k} / \rho(0)\right)^{N /\left(l_{0}+1\right)-N_{0}}} \frac{\left|E_{k}\right|^{\frac{1}{q}}}{\left|E_{j}\right|^{\frac{1}{q}}} \\
& \lesssim 2^{(k-j) n\left(\frac{1}{q}\right)}\left\|f_{j}\right\|_{L^{q}\left(\mathbb{R}^{n}\right)} .
\end{aligned}
$$


For $W_{3,2}$, using Hölder's inequality, we can easily get

$$
\begin{aligned}
W_{3,2} & \lesssim \frac{\left\|f_{j}\right\|_{L^{q}\left(\mathbb{R}^{n}\right)}}{\left(1+2^{k} / \rho(0)\right)^{N /\left(l_{0}+1\right)}} \frac{\left|E_{k}\right|^{\frac{1}{q}}}{\left|E_{j}\right|^{\frac{1}{q}}} . \\
& \lesssim 2^{(k-j) n\left(\frac{1}{q}\right)}\left\|f_{j}\right\|_{L^{q}\left(\mathbb{R}^{n}\right)} .
\end{aligned}
$$

Combining with $W_{31}$ and $W_{32}$, we get

$$
W_{3} \lesssim \sum_{k=-\infty}^{\infty} 2^{k \alpha p}\left(\sum_{j=-\infty}^{k-2} 2^{(k-j) n\left(\frac{1}{q}\right)}\left\|f_{j}\right\|_{L^{q}\left(\mathbb{R}^{n}\right)}\right)^{p}
$$

Since $-\frac{n}{q}<\alpha<n\left(1-\frac{1}{q}-\frac{1}{\tilde{q}}\right)$, Hölder's inequality and (4.1) imply that $W_{3} \lesssim\|f\|_{\dot{K}_{q}^{\alpha, p}}^{p}$. This completes the proof of Theorem 4.4.

Now we consider the boundedness of the commutators $\left[b, R_{L}\right]$ on Herz spaces, where $b \in \mathrm{BMO}_{\sigma}(\rho)$. If $s \geq n$ and $b \in \mathrm{BMO}\left(\mathbb{R}^{n}\right)$, the desired boundedness can be deduced from Theorem 1.1 in [29]. Hence in the following theorem, we still assume that $s \in(n / 2, n)$.

Theorem 4.5 Suppose that $V \in B_{s}, s>\frac{n}{2}$, and $b \in \mathrm{BMO}_{\sigma}(\rho), 0<\sigma<\infty$. Let $\left(\frac{1}{s}-\frac{1}{n}\right)^{+}=\frac{1}{\tilde{q}}$. If $0<p<\infty,(\tilde{q})^{\prime}<q<\infty$, and $-\frac{n}{q}<\alpha<n\left(1-\frac{1}{q}-\frac{1}{\tilde{q}}\right)$, then

$$
\left\|\left[b, R_{L}\right] f\right\|_{\dot{K}_{q}^{\alpha, p}\left(\mathbb{R}^{n}\right)} \lesssim\|f\|_{\dot{K}_{q}^{\alpha, p}\left(\mathbb{R}^{n}\right)} .
$$

Proof Similar to Theorem 4.4, we write

$$
f(y)=\sum_{j=-\infty}^{\infty} f(y) \chi_{E_{j}}(y)=\sum_{j=-\infty}^{\infty} f_{j}(y)
$$

Hence, we have

$$
\begin{aligned}
\left\|\left[b, R_{L}\right] f\right\|_{\dot{K}_{q}^{\alpha, p}\left(\mathbb{R}^{n)}\right.}^{p} \lesssim & \sum_{k=-\infty}^{\infty} 2^{k \alpha p}\left(\sum_{j=-\infty}^{k-2}\left\|\chi_{k}\left[b, R_{L}\right] f_{j}\right\|_{L^{q}\left(\mathbb{R}^{n}\right)}\right)^{p} \\
& +\sum_{k=-\infty}^{\infty} 2^{k \alpha p}\left(\sum_{j=k-1}^{k+1}\left\|\chi_{k}\left[b, R_{L}\right] f_{j}\right\|_{L^{q}\left(\mathbb{R}^{n)}\right.}\right)^{p} \\
& +\sum_{k=-\infty}^{\infty} 2^{k \alpha p}\left(\sum_{j=k+2}^{\infty}\left\|\chi_{k}\left[b, R_{L}\right] f_{j}\right\|_{L^{q}\left(\mathbb{R}^{n}\right)}\right)^{p} \\
= & \widetilde{W}_{1}+\widetilde{W}_{2}+\widetilde{W}_{3} .
\end{aligned}
$$

For $\widetilde{W}_{2}$, by Lemma 4.3 , we have

$$
\widetilde{W}_{2} \lesssim \sum_{k=-\infty}^{\infty} 2^{k \alpha p}\left(\sum_{j=k-1}^{k+1}\left\|f_{j}\right\|_{L^{q}\left(\mathbb{R}^{n}\right)}\right)^{p} \lesssim\|f\|_{K_{q}^{\alpha, p}\left(\mathbb{R}^{n}\right)}^{p}
$$


Now we estimate $\widetilde{W}_{1}$. For $x \in E_{k}, y \in E_{j}$ with $j \leq k-2,|x-y| \sim 2^{k}$. By Lemma 2.16, we can get

$$
\begin{aligned}
& \left\|\chi_{k}\left[b, R_{L}\right] f_{j}\right\|_{L^{q}\left(\mathbb{R}^{n}\right)} \\
& =\left(\int_{E_{k}}\left|\int_{E_{j}}(b(x)-b(y)) K_{R_{L}}(x, y) f(y) d y\right|^{q} d x\right)^{\frac{1}{q}} \\
& \lesssim \\
& \quad\left(\int_{E_{k}}\left|\int_{E_{j}} \frac{|(b(x)-b(y)) f(y)|}{(1+|x-y| / \rho(x))^{N}} \frac{1}{|x-y|^{n-1}} \int_{B(y,|x-y|)} \frac{V(z)}{|z-y|^{n-1}} d z d y\right|^{q} d x\right)^{\frac{1}{q}} \\
& \quad+\left(\int_{E_{k}}\left|\int_{E_{j}} \frac{|(b(x)-b(y)) f(y)|}{(1+|x-y| / \rho(x))^{N}} \frac{1}{|x-y|^{n}} d y\right|^{q} d x\right)^{\frac{1}{q}} \\
& =\widetilde{W}_{1,1}+\widetilde{W}_{1,2} .
\end{aligned}
$$

For $\widetilde{W}_{1,1}$, we can see that $b(x)-b(y)=\left(b(x)-b_{B_{j}}\right)-\left(b(y)-b_{B_{j}}\right)$. Hence, by Lemma 2.17 , we have

$$
\begin{aligned}
\widetilde{W}_{1,1} \lesssim & \frac{1}{\left(1+2^{k} / \rho(0)\right)^{N / l_{0}+1}} \\
& \times \frac{1}{\left|2^{k}\right|^{n-1}}\left(\int_{E_{k}}\left|\int_{E_{j}}\right|(b(x)-b(y)) f(y)\left|\int_{B(y,|x-y|)} \frac{V(z)}{|z-y|^{n-1}} d z d y\right|^{q} d x\right)^{\frac{1}{q}} \\
\lesssim & \frac{1}{\left(1+2^{k} / \rho(0)\right)^{N / l_{0}+1}} \frac{1}{\left|2^{k}\right|^{n-1}}\left(\int_{E_{k}}\left|\left(b(x)-b_{B_{j}}\right)\right|^{q} d x\right)^{\frac{1}{q}} \\
& \times \int_{E_{j}}|f(y)| \int_{B\left(0,2^{k+1}\right)} \frac{V(z)}{|z-y|^{n-1}} d z d y \\
& +\frac{1}{\left(1+2^{k} / \rho(0)\right)^{N / l_{0}+1}} \frac{\left|E_{k}\right|^{\frac{1}{q}}}{\left|2^{k}\right|^{n-1}} \int_{E_{j}}\left|\left(b(y)-b_{B_{j}}\right) f(y)\right| \\
& \times \int_{B\left(0,2^{k+1}\right)} \frac{V(z)}{|z-y|^{n-1}} d z d y \\
= & \widetilde{W}_{1,1}^{(1)}+\widetilde{W}_{1,1}^{(2)} .
\end{aligned}
$$

Using Hölder's inequality and the boundedness of the fractional integral $\mathcal{I}_{1}$ with $\frac{1}{\tilde{q}}=\frac{1}{s}-\frac{1}{n}$, we obtain

$$
\begin{aligned}
& \int_{E_{j}}\left|\left(b(y)-b_{B_{j}}\right) f(y)\right| \int_{B\left(0,2^{k+1}\right)} \frac{V(z)}{|z-y|^{n-1}} d z d y \\
& \quad \lesssim \frac{\|b\|_{\sigma}\left|E_{j}\right|^{1-\frac{1}{q}-\frac{1}{\tilde{q}}}}{\left(1+2^{j} / \rho(0)\right)^{-\left(l_{0}+1\right) \sigma}}\left\|f_{j}\right\|_{L^{q}\left(\mathbb{R}^{n}\right)}\left\|\mathcal{I}_{1}\left(V \chi_{B\left(0,2^{k+1}\right)}\right)\right\|_{L^{\tilde{q}}\left(\mathbb{R}^{n}\right)} \\
& \quad \lesssim \frac{\|b\|_{\sigma}\left|E_{j}\right|^{1-\frac{1}{q}-\frac{1}{\tilde{q}}}}{\left(1+2^{j} / \rho(0)\right)^{-\left(l_{0}+1\right) \sigma}}\left\|f_{j}\right\|_{L^{q}\left(\mathbb{R}^{n}\right)}\left\|V \chi_{B\left(0,2^{k+1}\right)}\right\|_{L^{s}\left(\mathbb{R}^{n}\right)} \\
& \quad \lesssim \frac{\|b\|_{\sigma}\left\|f_{j}\right\|_{L^{q}\left(\mathbb{R}^{n}\right)}}{\left(1+2^{k} / \rho(0)\right)^{-\left(l_{0}+1\right) \sigma-N_{0}}}\left|E_{j}\right|^{1-\frac{1}{q}-\frac{1}{\tilde{q}}}\left|E_{k}\right|^{\frac{1}{s^{-}-\frac{2}{n}}},
\end{aligned}
$$


where in the last inequality we have used (4.2) above. We get

$$
\widetilde{W}_{1,1}^{(2)} \lesssim \frac{\|b\|_{\sigma}\left\|f_{j}\right\|_{L^{q}\left(\mathbb{R}^{n}\right)}}{\left(1+2^{k} / \rho(0)\right)^{N /\left(l_{0}+1\right)-\left(l_{0}+1\right) \sigma-N_{0}}} \frac{\left|E_{j}\right|^{1-\frac{1}{q}-\frac{1}{\bar{q}}}}{\left|E_{k}\right|^{1-\frac{1}{q}-\frac{1}{\bar{q}}}} .
$$

Similar to $\widetilde{W}_{1,1}^{(2)}$, we have

$$
\int_{E_{j}}|f(y)| \int_{B\left(0,2^{k+1}\right)} \frac{V(z)}{|z-y|^{n-1}} d z d y \lesssim\left\|f_{j}\right\|_{L^{q}\left(\mathbb{R}^{n}\right)}\left(1+2^{k} / \rho(0)\right)^{N_{0}}\left|E_{j}\right|^{1-\frac{1}{q}-\frac{1}{\tilde{q}}}\left|E_{k}\right|^{\frac{1}{s}-\frac{2}{n}}
$$

By Lemma 2.10, we get

$$
\widetilde{W}_{1,1}^{(1)} \lesssim \frac{(k-j)\|b\|_{\sigma}\left\|f_{j}\right\|_{L^{q}\left(\mathbb{R}^{n}\right)}}{\left(1+2^{k} / \rho(0)\right)^{N /\left(l_{0}+1\right)-\left(l_{0}+1\right) \sigma-N_{0}}} \frac{\left|E_{j}\right|^{1-\frac{1}{q}-\frac{1}{q}}}{\left|E_{k}\right|^{1-\frac{1}{q}-\frac{1}{\bar{q}}}} .
$$

Since $N$ is large enough, when $j \leq k-2$, we have

$$
\widetilde{W}_{1,1} \lesssim(k-j) 2^{(j-k) n\left(1-\frac{1}{q}-\frac{1}{\tilde{q}}\right)}\|b\|_{\sigma}\left\|f_{j}\right\|_{L^{q}\left(\mathbb{R}^{n}\right)}
$$

Next we estimate $\widetilde{W}_{12}$. We can get

$$
\begin{aligned}
\widetilde{W}_{1,2} \lesssim & \frac{1}{\left(1+2^{k} / \rho(0)\right)^{N /\left(l_{0}+1\right)}} \frac{1}{\left|2^{k}\right|^{n}}\left(\int_{E_{k}}\left|\left(b(x)-b_{B_{j}}\right)\right|^{q} d x\right)^{\frac{1}{q}} \int_{E_{j}}|f(y)| d y \\
& +\frac{1}{\left(1+2^{k} / \rho(0)\right)^{N /\left(l_{0}+1\right)}} \frac{\left|E_{k}\right|^{\frac{1}{q}}}{\left|2^{k}\right|^{n}} \int_{E_{j}}\left|\left(b(y)-b_{B_{j}}\right) f(y)\right| d y \\
= & : \widetilde{W}_{1,2}^{(1)}+\widetilde{W}_{1,2}^{(2)} .
\end{aligned}
$$

Using Lemma 2.10 and the Hölder inequality, we get the following estimates:

$$
\widetilde{W}_{1,2}^{(1)} \lesssim \frac{(k-j)\|b\|_{\sigma}\left\|f_{j}\right\|_{L^{q}\left(\mathbb{R}^{n}\right)}}{\left(1+2^{k} / \rho(0)\right)^{N /\left(l_{0}+1\right)-\left(l_{0}+1\right) \sigma}} \frac{\left|E_{j}\right|^{1-\frac{1}{q}}}{\left|E_{k}\right|^{1-\frac{1}{q}}}
$$

and

$$
\widetilde{W}_{1,2}^{(2)} \lesssim \frac{\|b\|_{\sigma}\left\|f_{j}\right\|_{L^{q}\left(\mathbb{R}^{n}\right)}}{\left(1+2^{k} / \rho(0)\right)^{N /\left(l_{0}+1\right)-\left(l_{0}+1\right) \sigma}} \frac{\left|E_{j}\right|^{1-\frac{1}{q}}}{\left|E_{k}\right|^{1-\frac{1}{q}}} .
$$

Take $N$ large enough. Because $j \leq k-2$, we can see that

$$
\widetilde{W}_{1,2} \lesssim(k-j) 2^{(j-k) n\left(1-\frac{1}{q}-\frac{1}{\tilde{q}}\right)}\|b\|_{\sigma}\left\|f_{j}\right\|_{L^{q}\left(\mathbb{R}^{n}\right)}
$$

Hence,

$$
\left\|\chi_{k}\left[b, R_{L}\right] f_{j}\right\|_{L^{q}\left(\mathbb{R}^{n}\right)} \lesssim(k-j) 2^{(j-k) n\left(1-\frac{1}{q}-\frac{1}{\tilde{q}}\right)}\|b\|_{\sigma}\left\|f_{j}\right\|_{L^{q}\left(\mathbb{R}^{n}\right)} .
$$


Since $-\frac{n}{q}<\alpha<n\left(1-\frac{1}{q}-\frac{1}{\tilde{q}}\right)$, similar to Theorem 3.10, we use Hölder's inequality to obtain

$$
\begin{aligned}
\widetilde{W}_{1} & \lesssim \sum_{k=-\infty}^{\infty} 2^{k \alpha p}\left(\sum_{j=-\infty}^{k-2}(k-j) 2^{(j-k) n\left(1-\frac{1}{q}-\frac{1}{\tilde{q}}\right)}\|b\|_{\sigma}\left\|f_{j}\right\|_{L^{q\left(\mathbb{R}^{n}\right)}}\right)^{p} \\
& \lesssim\|b\|_{\sigma}^{p}\|f\|_{\dot{K}_{q}^{\alpha, p}}^{p}
\end{aligned}
$$

For $\widetilde{W}_{3}$, if $x \in E_{k}$ and $y \in E_{j}$ with $j \geq k+2$, then $|x-y| \sim 2^{j}$. We have

$$
\begin{aligned}
& \left\|\chi_{k}\left[b, R_{L}\right] f_{j}\right\|_{L^{q}\left(\mathbb{R}^{n}\right)} \\
& \lesssim\left(\int_{E_{k}}\left|\int_{E_{j}} \frac{|(b(x)-b(y)) f(y)|}{(1+|x-y| / \rho(x))^{N}} \frac{1}{|x-y|^{n-1}} \int_{B(y,|x-y|)} \frac{V(z)}{|z-y|^{n-1}} d z d y\right|^{q} d x\right)^{\frac{1}{q}} \\
& \quad+\left(\int_{E_{k}}\left|\int_{E_{j}} \frac{|(b(x)-b(y)) f(y)|}{(1+|x-y| / \rho(x))^{N}} \frac{1}{|x-y|^{n}} d y\right|^{q} d x\right)^{\frac{1}{q}} \\
& =: \widetilde{W}_{3,1}+\widetilde{W}_{3,2} .
\end{aligned}
$$

Using the decomposition $b(x)-b(y)=\left(b(x)-b_{B_{k}}\right)-\left(b(y)-b_{B_{k}}\right)$ again, we get

$$
\begin{aligned}
& \int_{E_{j}}\left|\left(b(y)-b_{B_{k}}\right) f(y)\right| \int_{B(y,|x-y|)} \frac{V(z)}{|z-y|^{n-1}} d z \\
& \quad \lesssim \int_{E_{j}}\left|\left(b(y)-b_{B_{k}}\right) f(y)\right| \int_{B\left(0,2^{j+1}\right)} \frac{V(z)}{|z-y|^{n-1}} d z d y \\
& \lesssim \frac{(k-j)\|b\|_{\sigma}\left\|f_{j}\right\|_{L^{q}\left(\mathbb{R}^{n}\right)}}{\left(1+2^{j} / \rho(0)\right)^{-\left(l_{0}+1\right) \sigma-N_{0}}}\left|E_{j}\right|^{1-\frac{1}{q}-\frac{1}{\tilde{q}}}\left|E_{j}\right|^{\frac{1}{s}-\frac{2}{n}}
\end{aligned}
$$

On the other hand,

$$
\int_{E_{j}}|f(y)| \int_{B\left(0,2^{j+1}\right)} \frac{V(z)}{|z-y|^{n-1}} d z d y \lesssim\left\|f_{j}\right\|_{L^{q}\left(\mathbb{R}^{n}\right)}\left(1+2^{j} / \rho(0)\right)^{N_{0}}\left|E_{j}\right|^{1-\frac{1}{q}-\frac{1}{\tilde{q}}}\left|E_{j}\right|^{\frac{1}{s}-\frac{2}{n}}
$$

Therefore

$$
\widetilde{W}_{3,1} \lesssim(j-k) 2^{(k-j) n\left(\frac{1}{q}\right)}\|b\|_{\sigma}\left\|f_{j}\right\|_{L^{q\left(\mathbb{R}^{n}\right)}}
$$

Similarly, we can get

$$
\widetilde{W}_{3,2} \lesssim(j-k) 2^{(k-j) n\left(\frac{1}{q}\right)}\|b\|_{\sigma}\left\|f_{j}\right\|_{L^{q}\left(\mathbb{R}^{n}\right)}
$$

Because $-\frac{n}{q}<\alpha<n\left(1-\frac{1}{q}-\frac{1}{\tilde{q}}\right)$, by the estimates for $\widetilde{W}_{31}$ and $\widetilde{W}_{33}$, we can use Hölder's inequality and (4.1) to get

$$
\widetilde{W}_{3} \lesssim \sum_{k=-\infty}^{\infty} 2^{k \alpha p}\left(\sum_{j=-\infty}^{k-2}(j-k) 2^{(k-j) n\left(\frac{1}{q}\right)}\|b\|_{\sigma}\left\|f_{j}\right\|_{L^{q}\left(\mathbb{R}^{n}\right)}\right)^{p} \lesssim\|b\|_{\sigma}^{p}\|f\|_{\dot{K}_{q}^{\alpha, p}}^{p} .
$$

This completes the proof of Theorem 4.5. 
Corollary 4.6 Suppose that $V \in B_{s}, s>\frac{n}{2}$, and $\left(\frac{1}{s}-\frac{1}{n}\right)^{+}=\frac{1}{\tilde{q}}$. Let $b \in \operatorname{BMO}_{\sigma}(\rho), 0<\sigma<\infty$. If $0<p<\infty, 1<q<\tilde{q},-\frac{n}{q}+\frac{n}{\tilde{q}}<\alpha<n\left(1-\frac{1}{q}\right)$, then:

(i) $\left\|R_{L}^{\prime} f\right\|_{\dot{K}_{q}^{\alpha, p}\left(\mathbb{R}^{n}\right)} \lesssim\|f\|_{\dot{K}_{q}^{\alpha, p}\left(\mathbb{R}^{n}\right)}$;

(ii) $\left\|\left[b, R_{L}^{\prime}\right] f\right\|_{\dot{K}_{q}^{\alpha, p}\left(\mathbb{R}^{n}\right)} \lesssim\|b\|_{\sigma}\|f\|_{\dot{K}_{q}^{\alpha, p}\left(\mathbb{R}^{n}\right)}$.

There are similar results for the non-homogeneous Herz space $K_{q}^{\alpha, p}\left(\mathbb{R}^{n}\right)$.

Theorem 4.7 Suppose that $V \in B_{s}, s>\frac{n}{2}$ and let $b \in \mathrm{BMO}_{\sigma}(\rho), 0<\sigma<\infty$. Let $0<p<\infty$, $\left(\frac{1}{s}-\frac{1}{n}\right)^{+}=\frac{1}{\tilde{q}}$.

(1) If $(\tilde{q})^{\prime}<q<\infty$ and $-\frac{n}{q}<\alpha<n\left(1-\frac{1}{q}-\frac{1}{\tilde{q}}\right)$, then

$$
\left\{\begin{array}{l}
\left\|R_{L} f\right\|_{K_{q}^{\alpha, p}\left(\mathbb{R}^{n}\right)} \lesssim\|f\|_{K_{q}^{\alpha, p}\left(\mathbb{R}^{n}\right)}, \\
\left\|\left[b, R_{L}\right] f\right\|_{K_{q}^{\alpha, p}\left(\mathbb{R}^{n}\right)} \lesssim\|b\|_{\sigma}\|f\|_{K_{q}^{\alpha, p}\left(\mathbb{R}^{n}\right)} .
\end{array}\right.
$$

(2) If $1<q<\tilde{q}$ and $-\frac{n}{q}+\frac{n}{\tilde{q}}<\alpha<n\left(1-\frac{1}{q}\right)$, then

$$
\left\{\begin{array}{l}
\left\|R_{L}^{\prime} f\right\|_{K_{q}^{\alpha, p}\left(\mathbb{R}^{n}\right)} \lesssim\|f\|_{K_{q}^{\alpha, p}\left(\mathbb{R}^{n}\right)}, \\
\left\|\left[b, R_{L}^{\prime}\right] f\right\|_{K_{q}^{\alpha, p}\left(\mathbb{R}^{n}\right)} \lesssim\|b\|_{\sigma}\|f\|_{K_{q}^{\alpha, p}\left(\mathbb{R}^{n}\right)} .
\end{array}\right.
$$

\section{Competing interests}

The authors declare that they have no competing interests.

\section{Authors' contributions}

All authors read and approved the final manuscript.

\section{Author details}

'Department of Mathematics, Qingdao University, Qingdao, Shandong 266071, China. ${ }^{2}$ Department of Mathematics, Shantou University, Shantou, Guangdong 515063, China.

\section{Acknowledgements}

Project supported by NSFC No. 11171203, No. 11201280, No. 11571217; New Teacher's Fund for Doctor Stations, Ministry of Education No. 20114402120003, University Science and Technology Projects of Shandong Province No. J15LI15.

Received: 1 March 2016 Accepted: 7 June 2016 Published online: 07 July 2016

\section{References}

1. Shen, Z: $L^{p}$ estimate for Schrödinger operator with certain potentials. Ann. Inst. Fourier 45, 513-546 (1995)

2. Sugano, S: Estimates for the operators $V^{\alpha}(-\Delta+V)^{-\beta}$ and $V^{\alpha} \nabla(-\Delta+V)^{-\beta}$ with certain non-negative potentials $V$. Tokyo J. Math. 21, 441-452 (1998)

3. Tang, L, Dong, J: Boundedness for some Schrödinger type operators on Morrey spaces related to certain nonnegative potentials. J. Math. Anal. Appl. 355, 101-109 (2009)

4. Yang, D, Yang, D, Zhou, Y: Endpoint properties of localized Riesz transforms and fractional integrals associated to Schrödinger operators. Potential Anal. 30, 271-300 (2009)

5. Jiang, Y: Endpoint estimates for fractional integral associated to Schrödinger operators on the Heisenberg groups. Acta Math. Sci. Ser. B 31, 993-1000 (2011)

6. Liu, Y: The weighted estimates for the operators $V^{\alpha}\left(-\Delta_{G}+V\right)^{-\beta}$ and $V^{\alpha} \nabla_{G}\left(-\Delta_{G}+V\right)^{-\beta}$ on the stratified Lie group $G$. J. Math. Anal. Appl. 349, 235-244 (2009)

7. Liu, Y, Dong, J: Some estimates of higher order Riesz transform related to Schrödinger type operators. Potential Anal. 32, 41-55 (2010)

8. Sugano, S: $L^{p}$ estimates for some Schrödinger operators and a Calderón-Zygmund operator of Schrödinger type. Tokyo J. Math. 30, 179-197 (2007)

9. Guo, Z, Li, P, Peng, L: $L^{p}$ boundedness of commutators of Riesz transforms associated to Schrödinger operator. J. Math. Anal. Appl. 341, 421-432 (2008)

10. Bui, T-A: Weighted estimates for commutators of some singular integrals related to Schrödinger operators. Bull. Sci. Math. 138, 270-292 (2014)

11. Liu, Y, Huang, J, Dong, J: Commutators of Calderón-Zygmund operators related to admissible functions on spaces of homogeneous type and applications to Schrödinger operators. Sci. China Math. 56, 1895-1913 (2013)

12. Liu, Y, Wang, L, Dong, C: Commutators of higher order Riesz transform associated with Schrödinger operators J. Funct. Spaces Appl. 2013, Article ID 842375 (2013) 
13. Wang, Y, Liu, Y: Higher order commutators of Riesz transforms related to Schrödinger operators. J. Inequal. Appl. $2014,466(2014)$

14. Bongioanni, B, Harboure, E, Salinas, O: Commutators of Riesz transforms related to Schrödinger operator. J. Fourier Anal. Appl. 17, 115-134 (2011)

15. Shen, Z: On the Neumann problem for Schrödinger operators in Lipschitz domains. Indiana Univ. Math. J. 43, 143-176 (1994)

16. John, F, Nirenberg, L: On function of bounded mean oscillation. Commun. Pure Appl. Math. 14, 415-426 (1961)

17. Liu, Y: Commutators of $B M O$ functions and degenerate Schrödinger operators with certain nonnegative potentials. Monatshefte Math. 165, 41-56 (2012)

18. Yang, D, Yang, D, Zhou, Y: Localized BMO and BLO spaces on RD-spaces and applications to Schrödinger operators. Commun. Pure Appl. Anal. 9, 779-812 (2010)

19. Herz, C: Lipschitz spaces and Bernstein's theorem on absolutely convergent Fourier transforms. J. Math. Mech. 18, 283-323 (1968/69)

20. Lu, S, Yang, D, Hu, G: Herz Type Spaces and Their Applications. Science Press, Beijing (2008)

21. Chen, W, Yang, D, Zhou, Z: Singular integrals and commutators in parabolic Herz spaces and their applications. Northeast. Math. J. 14, 440-454 (1998)

22. $\mathrm{Hu}, \mathrm{G}, \mathrm{Lu}, \mathrm{S}$, Yang, D: Boundedness of rough singular integral operators on homogeneous Herz spaces. J. Aust. Math. Soc. Ser. A 66, 201-223 (1999)

23. Tang, L, Yang, D: Boundedness of multilinear operators in Herz-type Hardy space. Acta Math. Sin. 16, 295-306 (2000)

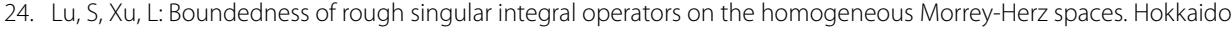
Math. J. 34, 299-314 (2005)

25. Dziubański, J, Zienkiewicz, J: $H^{p}$ spaces for Schrödinger operator, Fourier analysis and related topics. Banach Cent. Publ. 56, 45-53 (2002)

26. Dziubański, J, Zienkiewicz, J: Hardy spaces $H^{1}$ associated to Schrödinger operator with potential satisfying reverse Hölder inequality. Rev. Mat. Iberoam. 15, 279-296 (1999)

27. Coifman, R, Rochberg, R, Weiss, G: Factorization theorems for Hardy space in several variable. Ann. Math. 103, 611-635 (1988)

28. Li, P, Wan, X, Zhang, C: Schrödinger operators on generalized Morrey spaces. arXiv:1504.05075

29. Lu, S, Yang, D: The continuity of commutators on Herz type spaces. Mich. Math. J. 44, 255-281 (1997)

\section{Submit your manuscript to a SpringerOpen ${ }^{\circ}$ journal and benefit from:}

$\rightarrow$ Convenient online submission

Rigorous peer review

- Immediate publication on acceptance

- Open access: articles freely available online

- High visibility within the field

- Retaining the copyright to your article 